\title{
GROUND STATES OF BI-HARMONIC EQUATIONS WITH CRITICAL EXPONENTIAL GROWTH INVOLVING CONSTANT AND TRAPPING POTENTIALS
}

\author{
LU CHEN, GUOZHEN LU AND MAOCHUN ZHU
}

\begin{abstract}
In this paper, we first give a necessary and sufficient condition for the boundedness and the compactness for a class of nonlinear functionals in $H^{2}\left(\mathbb{R}^{4}\right)$. Using this result and the principle of symmetric criticality, we can present a relationship between the existence of the nontrivial solutions to the semilinear bi-harmonic equation of the form

$$
(-\Delta)^{2} u+\gamma u=f(u) \text { in } \mathbb{R}^{4}
$$

and the range of $\gamma \in \mathbb{R}^{+}$, where $f(s)$ is the general nonlinear term having the critical exponential growth at infinity.

Though the existence of the nontrivial solutions for the bi-harmonic equation with the critical exponential growth has been studied in the literature, it seems that nothing is known so far about the existence of the ground-state solutions for this class of equations involving the trapping potential introduced by Rabinowitz in [44. Since the trapping potential is not necessarily symmetric, classical radial method cannot be applied to solve this problem. In order to overcome this difficulty, we first establish the existence of the ground-state solutions for the equation
\end{abstract}

$$
\left.(-\Delta)^{2} u+V(x) u=\lambda s \exp \left(2|s|^{2}\right)\right) \text { in } \mathbb{R}^{4},
$$

when $V(x)$ is a positive constant using the Fourier rearrangement and the Pohozaev identity. Then we will explore the relationship between the Nehari manifold and the corresponding limiting Nehari manifold to derive the existence of the ground state solutions for the equation (0.1) when $V(x)$ is the Rabinowitz type trapping potential, namely it satisfies

$$
0<V_{0}=\inf _{x \in \mathbb{R}^{4}} V(x)<\lim _{|x| \rightarrow \infty} V(x)<+\infty .
$$

The same result and proof applies to the harmonic equation with the critical exponential growth involving the Rabinowitz type trapping potential in $\mathbb{R}^{2}$.

Keywords: Rabinowitz potential, Ground state solutions; Bi-harmonic equations; Adams' inequalities.

2010 MSC. Primary 46E35; 35J91; Secondary 26D10.

\section{INTRODUCTION}

The research of the second author was supported partly by the Simons Foundation. The research of the third author was supported by Natural Science Foundation of China (11601190), Natural Science Foundation of Jiangsu Province (BK20160483) and Jiangsu University Foundation Grant (16JDG043). 
Let $\Omega$ be an open domain in $\mathbb{R}^{n}$. We will consider the following nonlinear partial differential equation with critical growth

$$
(-\Delta)^{m} u=f(u) \text { in } \Omega \subset \mathbb{R}^{n},
$$

where $m=1$ or 2. Equations (1.1) have been extensively studied by many authors in bounded and unbounded domains.

In the case $n>2 m$, the subcritical and critical growth means that the nonlinearity cannot exceed the polynomial of degree $\frac{n+2 m}{n-2 m}$ by the Sobolev embedding. While in the case $n=2 m$, we say that $f(s)$ has critical exponential growth at infinity if there exists $\alpha_{0}>0$ such that

$$
\lim _{t \rightarrow \infty} \frac{f(t)}{\exp \left(\alpha t^{2}\right)}=\left\{\begin{array}{l}
0, \quad \text { for } \alpha>\alpha_{0} \\
+\infty, \text { for } \alpha<\alpha_{0}
\end{array}\right.
$$

The critical exponential growth in the case $m=1, n=2$ is given by the TrudingerMoser inequality ([41], [50]):

$$
\sup _{\substack{u \in H_{0}^{1}(\Omega) \\\|\nabla u\|_{L^{2}(\Omega)} \leq 1}} \int_{\Omega} e^{\alpha|u|^{2}} d x<\infty \text { iff } \alpha \leq 4 \pi,
$$

and in the case $m=2, n=4$ is given by the Adams inequality [2]:

$$
\sup _{\substack{u \in H_{0}^{2}(\Omega) \\\|\Delta u\|_{L^{2}(\Omega)} \leq 1}} \int_{\Omega} e^{\alpha|u|^{2}} d x<\infty \text { iff } \alpha \leq 32 \pi^{2} .
$$

The study of equation (1.1) with the critical exponential growth on bounded domain also involves a lack of compactness similar to the case $n>2 m$ at certain levels that the Palais-Smale compactness condition fails due to concentration phenomena. In order to overcome the possible failure of the Palais-Smale compactness condition, there is a common approach involved with the Trudinger-Moser and Adams type inequalities (see [13], [8], [16], 3], [22] and references therein).

If $\Omega$ is the entire Euclidean space $\mathbb{R}^{n}$, the earlier study of the existence of solutions for equation (1.1) with the critical exponential growth can date back to the work of Atkinson and Peletier [5, 4]. Indeed, the authors obtained the existence of ground state solutions for equation (1.1) by assuming that there exists some $y_{0}>0$ such that $g(t)=\log f(t)$ satisfies

$$
g^{\prime}(t)>0, g^{\prime \prime}(t) \geq 0
$$

for any $t \geq y_{0}$. This kind of growth condition allows us to take the nonlinearity $f(t)=$ $\left(t^{2}-t\right) \exp \left(t^{2}\right)$, which has critical exponential growth. In the literature, many authors have considered the existence of solutions for equations of the form

$$
(-\Delta)^{m} u+V(x) u=f(u) \text { in } \mathbb{R}^{n},
$$


where $n=2 m$, the nonlinearity $f(s)$ has critical exponential growth, and the potential $V(x)$ is bounded away from zero. For the equation (1.4), the loss of compactness may be produced not only by the concentration phenomena but also by the vanishing phenomena! We will describe some of the relevant works below.

When $V(x)$ is a coercive potential, that is,

$$
V(x) \geq V_{0}>0 \text {, and additionally either } \lim _{x \rightarrow \infty} V(x)=+\infty \text { or } \frac{1}{V} \in L^{1}\left(\mathbb{R}^{n}\right),
$$

the existence and multiplicity results of equation (1.4) can be found in the papers [52, [23], [53] and the references therein. Their proofs depend crucially on the compact embeddings given by the coercive potential, and the vanishing phenomena can be ruled out.

When $V(x)$ is the constant potential, i.e. $V(x)=V_{0}>0$, the natural space for a variational treatment of (1.4) is $H^{m}\left(\mathbb{R}^{n}\right)$. It is well known that the embedding $H^{m}\left(\mathbb{R}^{n}\right) \hookrightarrow$ $L^{2}\left(\mathbb{R}^{n}\right)$ is continuous but not compact, even in the radial case. In the case $m=2$, the existence of nontrivial solutions for equation (1.4) was obtained by Chen et al in [14] (see also [12]) (see [6] for $m=1$ ) under the assumptions that for any $p>2$,

$$
f(s) \geq \eta_{p} s^{p-1}, \forall s \geq 0,
$$

where $\eta_{p}$ is some constant depending on $p$, and by Sani in [49] (see [47] or [5] for $m=1$ ) under the assumption

$$
\lim _{s \rightarrow+\infty} \frac{s f(s)}{\exp \left(32 \pi^{2} s^{2}\right)} \geq \beta_{0}>0
$$

In their proofs, the so-called Trudinger-Moser-Adams inequality in the whole $\mathbb{R}^{2}$ or $\mathbb{R}^{4}$ plays a crucial role. Now, let's mention some of these inequalities. In 2000, AdachiTanaka [1] (see also do O [18]) obtained a sharp Trudinger-Moser inequality on $\mathbb{R}^{n}$ :

$$
\sup _{\substack{u \in W^{1, n}\left(\mathbb{R}^{n}\right), \int_{\mathbb{R}^{n}}|\nabla u|^{n} d x \leq 1}} \int_{\mathbb{R}^{n}} \Phi_{n}\left(\alpha|u|^{\frac{n}{n-1}}\right) d x \leq C(\alpha, n)\|u\|_{n}^{n}, \text { iff } 0<\alpha<\alpha_{n},
$$

where $\Phi_{n}(t):=e^{t}-\sum_{i=0}^{n-2} \frac{t^{i}}{i !}$. Note that the inequality (1.7) has the subcritical form, that is $\alpha<\alpha_{n}$. Later, in [46] and [32], Li and Ruf showed that the best exponent $\alpha_{n}$ becomes admissible if the Dirichlet norm $\int_{\mathbb{R}^{n}}|\nabla u|^{2} d x$ is replaced by Sobolev norm $\int_{\mathbb{R}^{n}}\left(|u|^{2}+|\nabla u|^{2}\right) d x$. More precisely, they proved that

$$
\sup _{\substack{u \in W^{1, n}\left(\mathbb{R}^{n}\right) \\ \int_{\mathbb{R}^{n}}\left(|u|^{n}+|\nabla u|^{n}\right) d x \leq 1}} \int_{\mathbb{R}^{2}} \Phi_{n}\left(\alpha|u|^{\frac{n}{n-1}}\right) d x<+\infty, \text { iff } \alpha \leq \alpha_{n} .
$$

The proofs of both the critical and subcritical Trudinger-Moser inequalities (1.7) and (1.8) rely on the Pólya-Szegö inequality and the symmetrization argument. Lam and $\mathrm{Lu}$ ([25], [24]) developed a symmetrization-free method to establish the critical Trudinger-Moser 
inequality (see also Lam, Lu and Tang [27] for a proof of the subcritical Trudinger-Moser inequality) in settings such as the Heisenberg group where the Pólya-Szegö inequality fails. Such an argument also provides an alternative proof of both critical and subcritical Trudinger-Moser inequalities (1.7) and (1.8) in the Euclidean space. In fact, the equivalence and relationship between the supremums of critical and subcritical Trudinger-Moser inequalities have been established by Lam, Lu and Zhang [28].

In 1995, Ozawa 42 obtained the Adams inequality in Sobolev space $W^{m, \frac{n}{m}}\left(\mathbb{R}^{n}\right)$ on the entire Euclidean space $\mathbb{R}^{n}$ by using the restriction $\left\|\Delta^{\frac{m}{2}} u\right\|_{\frac{n}{m}} \leq 1$. However, with the argument in [42, 21, one cannot obtain the best possible exponent $\beta$ for this type of inequality. Sharp Adams inequality in the case of even order of derivatives was proved by Ruf and Sani [48] under the constraint

$$
\left\{u \in W^{m, \frac{n}{m}} \mid\left\|(I-\Delta)^{\frac{m}{2}}\right\|_{\frac{n}{m}} \leq 1\right\},
$$

when $m$ is an even integer. When the order $m$ of the derivatives is odd, a sharp Adams inequality was established by Lam and Lu [26]. A uniform proof was given for all orders $m$ of derivatives including fractional orders of derivatives by Lam and Lu in [24] through a rearrangement-free argument.

The authors in 24] obtained the sharp Adams inequality under the Sobolev norm constraint:

$$
\sup _{\substack{u \in H^{2}\left(\mathbb{R}^{4}\right) \\
\|\Delta u\|_{2}^{2}+\|u\|_{2}^{2} \leq 1}} \int_{\mathbb{R}^{4}}\left(\exp \left(\beta|u(x)|^{2}\right)-1\right) d x\left\{\begin{array}{l}
\leq C \text { if } \beta \leq 32 \pi^{2} \\
=+\infty \text { if } \beta>32 \pi^{2}
\end{array}\right.
$$

In 2011, Ibrahim et al [19] discovered a sharpened Trudinger-Moser inequality on $\mathbb{R}^{2}$-the Trudinger-Moser inequality with the exact growth condition:

$$
\sup _{\substack{u \in H^{1}\left(\mathbb{R}^{2}\right) \\ \int_{\mathbb{R}^{4}}|\nabla u|^{2} d x \leq 1}} \int_{\mathbb{R}^{2}} \frac{\exp \left(4 \pi|u|^{2}\right)-1}{(1+|u|)^{p}} d x \leq C_{p} \int_{\mathbb{R}^{2}}|u|^{2} d x \text { iff } p \geq 2 .
$$

Later, (1.10) was extended to the general case $n \geq 3$ by Masmoudi and Sani [39] (see Lam et al [29] for inequalities with exact growth under different norms) and to the framework of hyperbolic space by Lu and Tang in [34]. It is interesting to notice that the TrudingerMoser inequality with the exact growth condition can imply both the inequalities (1.7) and (1.8).

The Adams' inequality with the exact growth condition was obtained by Masmoudi and Sani 38] in dimension 4:

$$
\sup _{\substack{u \in H^{2}\left(\mathbb{R}^{4}\right) \\ \int_{\mathbb{R}^{4}}|\Delta v|^{2} d x \leq 1}} \int_{\mathbb{R}^{4}} \frac{\exp \left(32 \pi^{2}|v|^{2}\right)-1}{(1+|v|)^{p}} d x \leq C_{p} \int_{\mathbb{R}^{4}}|v|^{2} d x \text { iff } p \geq 2,
$$


and then established in any dimension $n \geq 3$ by Lu et al in [35] (see [40] for higher order case). Further improvement of Adams inequalities can also be found in recent work of $\mathrm{Lu}$ and Yang [37] where sharpened Hardy-Adams inequalities were established in $\mathbb{R}^{4}$ using Fourier analysis on hyperbolic spaces (see also [31] for higher even dimensions).

Based on the Trudinger-Moser inequality with the exact growth, Ibrahim et al obtained a sufficient and necessary condition for compactness of general nonlinear functionals (see [39] for $n \geq 3$ ). This sufficient and necessary condition is a strong tool to study the existence of solutions for the semilinear equation under a very general assumption on the nonlinearity. Indeed, they consider the equations of the form:

$$
-\Delta u+\gamma u=f(u) \text { in } \mathbb{R}^{2},
$$

where $\gamma$ is a positive constant and $f(s)$ has the critical exponential growth at infinity. They establish the following result.

Proposition 1.1. If $f$ satisfies $f(0)=0$ and the conditions (1.2), (i) and (ii)(see Section 2), then there exists $\gamma^{*} \in(0,+\infty)$ such that for each $\gamma \in\left(0, \gamma^{*}\right)$, the equation admits a positive ground state solution.

The number $\gamma^{*}$ above is associated with the so called Trudinger-Moser ratio, and both the growth conditions (1.5) and (1.6) imply that the constant $\gamma$ appearing in (1.12) satisfies

$$
\gamma<\gamma^{*} \text {. }
$$

Their arguments depend crucially on the Pohozaev identity and Schwarz symmetrization argument.

Motivated by the results just described, in this paper, we are interested in the existence of ground state solutions for the biharmonic equations

$$
(-\Delta)^{2} u+V(x) u=f(u) \text { in } \mathbb{R}^{4},
$$

where the nonlinear term $f(s)$ has the critical exponential growth (1.2) at infinity and the potential $V(x)$ is bounded away from zero.

As far as we know, there are no related results about the existence of ground state solutions for the problem (1.13) by means of variational methods. In study of the problems involving the bi-harmonic operator, we will encounter many more difficulties than in the case for the Lapacian. For example, we cannot always rely on the maximum principle, and there is no Pólya-Szegö type inequality for the second order derivatives. Thus, we cannot use Schwarz symmetrization principle. Moreover, if $u$ belongs $H^{2}\left(\mathbb{R}^{4}\right)$, we cannot claim that $|u|, u^{+}$or $u^{-}$belong to $H^{2}\left(\mathbb{R}^{4}\right)$. Therefore, we cannot expect to obtain a positive solution. 


\section{The MAIN RESUlts}

In order to obtain the existence of solutions to the equation (1.13), we first establish the necessary and sufficient conditions for the boundedness and the compactness of general nonlinear functionals in $H^{2}\left(\mathbb{R}^{4}\right)$.

Theorem 2.1 (Boundedness). Suppose that $g: \mathbb{R} \rightarrow[0,+\infty)$ is a Borel function and define

$$
G(u)=\int_{\mathbb{R}^{4}} g(u) d x .
$$

Then for any $K>0$, the following conditions are equivalent

(1) $\lim _{t \rightarrow+\infty}|t|^{2} \exp \left(-\frac{1}{K}|t|^{2}\right) g(t)<\infty, \lim _{t \rightarrow 0}|t|^{-2} g(t)<\infty$.

(2) There exists a constant $C_{g, K}>0$ such that for any $u \in H^{2}\left(\mathbb{R}^{4}\right)$ satisfying $\int_{\mathbb{R}^{4}}|\Delta u|^{2} d x \leq$ $32 \pi^{2} K$, there holds

$$
\int_{\mathbb{R}^{4}} g(u) d x \leq C_{g, K} \int_{\mathbb{R}^{4}}|u|^{2} d x .
$$

Theorem 2.2 (Compactness). Suppose that $g: \mathbb{R} \rightarrow[0,+\infty)$ is a continuous function and define

$$
G(u)=\int_{\mathbb{R}^{4}} g(u) d x .
$$

Then for any $K>0$, the following conditions are equivalent

(3) $\lim _{t \rightarrow+\infty}|t|^{2} \exp \left(-\frac{1}{K}|t|^{2}\right) g(t)=0, \lim _{t \rightarrow 0}|t|^{-2} g(t)=0$.

(4) For any radially symmetric sequence $\left\{u_{k}\right\}_{k} \in H^{2}\left(\mathbb{R}^{4}\right)$ satisfying $\int_{\mathbb{R}^{4}}|\Delta u|^{2} d x \leq$ $32 \pi^{2} K$ and weakly converging to some $u$, we have that $G\left(u_{k}\right) \rightarrow G(u)$.

As an application, we study the following bi-harmonic equation with the constant potential,

$$
(-\Delta)^{2} u+\gamma u=f(u) \text { in } \mathbb{R}^{4}
$$

where the nonlinearity $f(t)$ is a continuous function on $\mathbb{R}$ satisfying (1.2), $f(0)=0$ and the following properties:

(i) (Ambrosetti-Rabinowitz condition) There exists $\mu>2$ such that $0<\mu F(t)=$ $\mu \int_{0}^{t} f(s) d s \leq t f(t)$ for any $t \in \mathbb{R}^{+}$

(ii) There exist $t_{0}$ and $M_{0}>0$ such that $F(t) \leq M_{0} f(t)$ for any $t \geq t_{0}$.

Theorem 2.3. Assume that $f$ satisfies $f(0)=0$ and the conditions (1.2), (i) and (ii), then there exists $\gamma^{*} \in(0,+\infty]$ such that for any $\gamma \in\left(0, \gamma^{*}\right)$, the equation (2.1) admits a non-trivial radial solution. Moreover, $\gamma^{*}$ is equal to the Admas ratio:

$$
C_{A}^{*}=\sup \left\{\frac{2}{\|u\|_{2}^{2}} \int_{\mathbb{R}^{4}} F(u) d x \mid u \in H_{r}^{2}\left(\mathbb{R}^{4}\right),\|\Delta u\|_{2}^{2} \leq \frac{32 \pi^{2}}{\alpha_{0}}\right\},
$$



where $H_{r}^{2}\left(\mathbb{R}^{4}\right)$ is the collection of all radial functions in $H^{2}\left(\mathbb{R}^{4}\right)$. In particular, $\gamma^{*}=+\infty$ is equivalent to

$$
\lim _{t \rightarrow \infty} \frac{t^{2} F(t)}{\exp \left(\alpha_{0} t^{2}\right)}=+\infty .
$$

Remark 2.4. If $F(t)=\frac{\exp \left(t^{2}\right)-1-t^{2}}{1+|t|^{\theta}}$, obviously, $f(t)=F^{\prime}(t)$ satisfies the conditions (1.2), (i) and (ii). By the Adams inequality with exact growth (1.11), we know that if $\theta<2$, then $\gamma^{*}=+\infty$, and the equation (2.1) admits a non-trivial radial solution for any $\gamma>0$. If $\theta \geq 2$, then $\gamma^{*}<+\infty$, and the equation (2.1) admits a non-trivial radial solution for any $\gamma \in\left(0, \gamma^{*}\right)$. Both the growth conditions of the nonlinearities used in [49] and [14] imply that the constant $\gamma$ appearing in Theorem 2.3 satisfies

$$
\gamma<\gamma^{*}
$$

Corollary 2.5. Assume that $f$ satisfies $f(0)=0$ and the conditions (1.2), (i), (ii), and $F(t)$ satisfies

$$
\lim _{t \rightarrow \infty} \frac{t^{2} F(t)}{\exp \left(\alpha_{0} t^{2}\right)}=+\infty,
$$

then for any $\gamma>0$, the equation (2.1) admits a non-trivial radial solution.

Until now, whether the solutions obtained in Theorem 2.3 and Corollary 2.5 are ground state solutions is unknown. However, if the nonlinearity has the special form $f(t)=$ $\lambda t \exp \left(2|t|^{2}\right)$, we can prove that the solutions obtained are ground-state solutions.

Theorem 2.6. For any $\gamma \in(0,+\infty)$, the equation

$$
(-\Delta)^{2} u+\gamma u=\lambda u \exp \left(2|u|^{2}\right) \text { in } \mathbb{R}^{4}
$$

admits a radial ground state solution if $\lambda \in(0, \gamma)$.

Based on the above Theorem, we can also obtain the existence of ground state solutions of the bi-harmonic equation with the Rabinowitz type potential, that is, the potential $V(x)$ is a continuous function satisfying

$$
0<\lambda<V_{0}=\inf _{x \in \mathbb{R}^{4}} V(x)<\lim _{|x| \rightarrow \infty} V(x)=\gamma<+\infty .
$$

This kind of potential was first introduced by Rabinowitz in [44].

Theorem 2.7. Assume that $V(x)$ is a continuous function satisfying (2.2), the equation

$$
(-\Delta)^{2} u+V(x) u=\lambda u \exp \left(2|u|^{2}\right) \text { in } \mathbb{R}^{4}
$$

admits a non-radial ground state solution. 
In general, the ground state solution can be constructed by showing that the infimum on the Pohozaev or Nehari manifold is achieved. This is equivalent to proving that the mountain-pass minimax level is achieved (see [8], [43], [45]). In the proof of Theorem [2.6, we cannot use the Schwarz symmetrization principle directly. In order to overcome this difficulty, we will apply the Fourier rearrangement proved by Lenzmann and Sok in [30] to obtain a radially minimizing sequence for the infimum on the Pohozaev manifold. While in the proof of Theorem 2.7, we will exploit the relationship between the Nehari manifold and the corresponding limiting Nehari manifold.

Using Proposition 1.1 and carrying out the same proof procedure of Theorem 2.7, we can also obtain the existence of ground state solutions of the following Laplacian equation with the Rabinowitz type potential introduced in [44]:

$$
-\Delta u+V(x) u=\lambda u \exp \left(|u|^{2}\right) \text { in } \mathbb{R}^{2} .
$$

Theorem 2.8. Assume that $V(x)$ is a continuous function satisfying

$$
0<\lambda<V_{0}=\inf _{x \in \mathbb{R}^{2}} V(x)<\lim _{|x| \rightarrow \infty} V(x)=\gamma<+\infty
$$

the equation (2.4) admits a non-radial ground state solution.

As far as we know, the Rabinowitz type potentials are only involved in the study of equations with the subcritical polynomial growth (see e.g., [33], [44] and [51]). In the case of $m=1, n=2$, when we replace the operator $-\Delta$ by $-\varepsilon^{2} \Delta$ in the above theorem when the nonlinear term has the exponential growth, the existence of semiclassical state $u_{\varepsilon}$ was obtained by Alves and Figueiredo in $\mathbb{R}^{2}$ [7] if $\varepsilon<<1$. For other related work on the semiclassical state of nonlinear Schrödinger equations in the case of subcritical nonlinear polynomial growth, we just name a few among a vast literature, e.g., [9, 11, 17, 20, 36, 51, the book [10] and many references therein. Nevertheless, as far as we are concerned, nothing is known if $\varepsilon=1$ and the nonlinear term has the exponential growth. Theorem 2.8 appears to be the first existence result for equation with the critical exponential growth involving the Rabinowitz type trapping potential.

This paper is organized as follows. Section 3 is devoted to the proof of the necessary and sufficient conditions for the boundedness and the compactness of general nonlinear functionals in $H^{2}\left(\mathbb{R}^{4}\right)$. In Section 4 , we will prove the existence of non-trivial solutions of the equation (2.1) with the constant potential under a very general assumption on the nonlinearity. In Section 5, we prove the existence of ground state solutions for the biharmonic equation (2.1) with the constant potential when the nonlinearity has the special form $f(s)=\lambda s \exp \left(2 s^{2}\right)$. In Section 6 , we prove the existence of ground state solutions for the bi-harmonic equation (2.1) with the Rabinowitz type potential.

Throughout this paper, the letter $c$ always denotes some positive constant which may vary from line to line. 
GROUND STATES OF BI-HARMONIC EQUATIONS WITH CRITICAL EXPONENTIAL GROWTH 9

\section{NECESSARY AND SUFFICIENT CONDITIONS FOR THE BOUNDEDNESS AND COMPACTNESS}

In this section, we will give the necessary and sufficient conditions for the boundedness and the compactness of general nonlinear functionals.

Proof of Theorems 2.1 and 2.2. Necessity of (1) in Theorem 2.1 and (3) in Theorem 2.2:

In order to prove the necessity of (1), we only need to verify that if (1) fails, then there exists a sequence $\left\{u_{k}\right\}_{k} \in H^{2}\left(\mathbb{R}^{4}\right)$ satisfying

$$
\int_{\mathbb{R}^{4}}\left|\Delta u_{k}\right|^{2} d x \leq 32 \pi^{2} K, \quad \int_{\mathbb{R}^{4}}\left|u_{k}\right|^{2} d x \rightarrow 0, \quad G\left(u_{k}\right) \rightarrow \infty .
$$

Similarly, in order to prove the necessity of (3), we only need to show that if (3) fails, then there exists a radially symmetric sequence $\left\{u_{k}\right\}_{k} \in H^{2}\left(\mathbb{R}^{4}\right)$ satisfying $\left\|\Delta u_{k}\right\|_{2}^{2} \leq 32 \pi^{2} K$ and weakly converging to 0 , such that $G\left(u_{k}\right)>\delta$ for some $\delta>0$.

First, we consider the case that the conditions (1) and (3) fails at the origin. Let $\left\{\phi_{k}\right\}_{k} \in$ $H^{2}\left(\mathbb{R}^{4}\right)$ be a sequence of spherically symmetric functions given by

$$
\phi_{k}(x)= \begin{cases}a_{k}, & \text { if } 0 \leq|x| \leq R_{k} \\ a_{k}\left(1-R_{k}^{2}-|x|^{2}+2 R_{k}|x|\right), & \text { if } R_{k}<|x| \leq R_{k}+1, \\ \eta_{k}(x), & \text { if }|x|>R_{k}+1,\end{cases}
$$

where $\eta_{k}$ is a smooth function satisfying

$$
\left.\eta_{k}(x)\right|_{\partial B_{R_{k}+1}}=0,\left.\quad \frac{\partial \eta_{k}(x)}{\partial \nu}\right|_{\partial B_{R_{k}+1}}=-2 a_{k},
$$

and

$$
\left.\eta_{k}(x)\right|_{\partial B_{R_{k}+2}}=0,\left.\quad \frac{\partial \eta_{k}(x)}{\partial \nu}\right|_{\partial B_{R_{k}+2}}=0 .
$$

Furthermore, we assume that $\left\{a_{k}\right\}_{k}$ and $\left\{R_{k}\right\}_{k}$ are positive sequences satisfying $\lim _{k \rightarrow \infty} a_{k}=$ 0 , and $\lim _{k \rightarrow \infty} R_{k}=\infty$.

Direct calculations show that there exists a constant $c>0$ such that

$$
\int_{\mathbb{R}^{4}}\left|\phi_{k}\right|^{2} d x \leq c a_{k}^{2} R_{k}^{4}, \int_{\mathbb{R}^{4}}\left|\Delta \phi_{k}\right|^{2} d x \leq c a_{k}^{2} R_{k}^{3}, \text { and } G\left(\phi_{k}\right) \geq \frac{\omega_{3}}{4} g\left(a_{k}\right) R_{k}^{4} .
$$

If (1) is violated by $\lim _{t \rightarrow 0}|t|^{-2} g(t)<\infty$, then there exists a sequence $c_{k} \rightarrow \infty$ such that $g\left(a_{k}\right) \geq c_{k} a_{k}^{2}$. Let $R_{k}=a_{k}^{-1 / 4}+a_{k}^{-1 / 2} c_{k}^{-1 / 8}$, then

$$
a_{k}^{2} R_{k}^{4} \rightarrow 0, G\left(u_{k}\right) \geq \frac{\omega_{3}}{4} c_{k} a_{k}^{2} R_{k}^{4} \rightarrow \infty
$$

If (3) is violated by $\lim _{t \rightarrow 0}|t|^{-2} g(t)>0$, then there exists $\delta>0$ such that $g\left(a_{k}\right) \geq \delta a_{k}^{2}$. Pick $R_{k}=a_{k}^{-1 / 2}$, then $a_{k}^{2} R_{k}^{4}=1, a_{k}^{2} R_{k}^{3} \rightarrow 0$ and

$$
G\left(u_{k}\right) \geq \frac{\omega_{3}}{4} g\left(a_{k}\right) R_{k}^{4} \geq \frac{\omega_{3}}{4} \delta>0 .
$$


What left is to consider the case when the conditions (1) and (3) do not hold at infinity. Let $\left\{b_{k}\right\}_{k} \subset \mathbb{R}^{+}, b_{k} \rightarrow \infty$, be such that

$$
\lim _{t \rightarrow+\infty}|t|^{2} \exp \left(-\frac{1}{K}|t|^{2}\right) g(t)=\lim _{k \rightarrow \infty} c_{k}
$$

where

$$
c_{k}:=b_{k}^{2} \exp \left(-\frac{1}{K} b_{k}^{2}\right) g\left(b_{k}\right) .
$$

Set $R_{k}=\exp \left(-\frac{1}{K} b_{k}^{2}\right)$, then $c_{k}=b_{k}^{2} R_{k} g\left(b_{k}\right)$.

Now, we consider the so-called Moser's sequence $\left\{\psi_{k}\right\}_{k} \in H^{2}\left(\mathbb{R}^{4}\right)$ consisting of spherically symmetric functions defined by

$$
\psi_{k}(x)= \begin{cases}b_{k}-\frac{2 K|x|^{2}}{R_{k}^{1 / 2} b_{k}}+\frac{2 K}{b_{k}}, & \text { if } 0 \leq|x| \leq R_{k}^{1 / 4} \\ \frac{4 K|\log | x||}{b_{k}}, & \text { if } R_{k}^{1 / 4}<|x| \leq 1 \\ \eta_{k}, & \text { if }|x|>1,\end{cases}
$$

where $\eta_{k}$ is a smooth function satisfying

$$
\left.\eta_{k}(x)\right|_{\partial B_{1}}=0,\left.\quad \frac{\partial \eta_{k}(x)}{\partial \nu}\right|_{\partial B_{1}}=\frac{4 K}{b_{k}}
$$

and

$$
\left.\eta_{k}(x)\right|_{\partial B_{2}}=0,\left.\quad \frac{\partial \eta_{k}(x)}{\partial \nu}\right|_{\partial B_{2}}=0 .
$$

Careful computations yield that there exists a constant $c>0$ such that

$$
\int_{\mathbb{R}^{4}}\left|\psi_{k}\right|^{2} d x \leq \frac{c K^{2}}{b_{k}^{2}}, \int_{\mathbb{R}^{4}}\left|\Delta \phi_{k}\right|^{2} d x=32 \pi^{2} K+O\left(\frac{1}{b_{k}^{2}}\right),
$$

and

$$
G\left(\psi_{k}\right) \geq \frac{\omega_{3}}{4} g\left(b_{k}\right) R_{k}=\frac{\omega_{3}}{4} \frac{c_{k}}{b_{k}^{2}}
$$

Define a new sequence $\left\{u_{k}\right\}_{k} \in H^{2}\left(\mathbb{R}^{4}\right)$ by $u_{k}(x)=\psi_{k}\left(x / S_{k}\right)$, then

$$
\int_{\mathbb{R}^{4}}\left|u_{k}\right|^{2} d x \leq \frac{c S_{k}^{4} K^{2}}{b_{k}^{2}}, \int_{\mathbb{R}^{4}}\left|\Delta u_{k}\right|^{2} d x=32 \pi^{2} K+O\left(\frac{1}{b_{k}^{2}}\right)
$$

and

$$
G\left(u_{k}\right)=S_{k}^{4} G\left(\psi_{k}\right) \geq \frac{\omega_{3}}{4} \frac{S_{k}^{4} c_{k}}{b_{k}^{2}} .
$$

Assume that the condition (1) does not hold at infinity, namely

$$
\lim _{t \rightarrow+\infty}|t|^{2} \exp \left(-\frac{1}{K}|t|^{2}\right) g(t)=\lim _{k \rightarrow \infty} c_{k}=\infty .
$$


Set $S_{k}^{4}=b_{k}^{2} c_{k}^{-1 / 2}$, there holds

$$
\int_{\mathbb{R}^{4}}\left|u_{k}\right|^{2} d x \leq \frac{c S_{k}^{4} K^{2}}{b_{k}^{2}} \rightarrow 0, \text { and } G\left(u_{k}\right) \geq \frac{\omega_{3}}{4} \frac{S_{k}^{4} c_{k}}{b_{k}^{2}} \rightarrow \infty .
$$

Assume that the condition (3) fails at infinity, namely, there exist some $\delta>0$ such that

$$
\lim _{t \rightarrow+\infty}|t|^{2} \exp \left(-\frac{1}{K}|t|^{2}\right) g(t)=\lim _{k \rightarrow \infty} c_{k}=\delta>0 .
$$

Set $S_{k}^{4}=b_{k}^{2}$, we can easily verify that $u_{k} \rightarrow 0$ a.e. $\mathbb{R}^{4}$, and

$$
\int_{\mathbb{R}^{4}}\left|u_{k}\right|^{2} d x \leq c K^{2}, \int_{\mathbb{R}^{4}}\left|\Delta u_{k}\right|^{2} d x=32 \pi^{2} K+O\left(\frac{1}{b_{k}^{2}}\right) .
$$

Moreover, we also have $u_{k} \rightarrow 0$ a.e. $\mathbb{R}^{4}$ and

$$
G\left(u_{k}\right) \geq \frac{\omega_{3}}{4} \frac{S_{k}^{4} \delta}{b_{k}^{2}}=\frac{\omega_{3} \delta}{4}>0 .
$$

This accomplish the proof of the necessity of (1) and (3).

Sufficiency of (1) and (2):

We first prove that (1) can imply (2). Define a new Borel measurable function $\tilde{g}(t)$ by $\tilde{g}(t)=g\left(\left(32 \pi^{2} K\right)^{\frac{1}{2}} t\right)$. Obviously,

$$
\lim _{t \rightarrow+\infty}|t|^{2} \exp \left(-32 \pi^{2}|t|^{2}\right) \tilde{g}(t)<\infty, \text { and } \lim _{t \rightarrow 0}|t|^{-2} \tilde{g}(t)<\infty
$$

By the Adams' inequality (1.11) with the exact growth in $\mathbb{R}^{4}$, we derive that

$$
\int_{\mathbb{R}^{4}} \tilde{g}(u) d x \leq c \int_{\mathbb{R}^{4}} \frac{\Phi\left(32 \pi^{2}|u|^{2}\right)}{(1+|u|)^{2}} d x \leq c \int_{\mathbb{R}^{4}}|u|^{2} d x
$$

Let $v=\left(32 \pi^{2} K\right)^{-1 / 2} u$. Then for any $u \in H^{2}\left(\mathbb{R}^{4}\right)$ satisfying $\|\Delta u\|_{2}^{2} \leq 32 \pi^{2} K$, there holds

$$
\int_{\mathbb{R}^{4}} g(u) d x=\int_{\mathbb{R}^{4}} \tilde{g}(v) d x \leq c \int_{\mathbb{R}^{4}}|v|^{2} d x \leq c \int_{\mathbb{R}^{4}}|u|^{2} d x .
$$

Now, we turn to prove the sufficiency of (3). Let $g: \mathbb{R} \rightarrow[0,+\infty)$ be a continuous function satisfying

$$
\lim _{t \rightarrow+\infty}|t|^{2} \exp \left(-\frac{|t|^{2}}{K}\right) g(t)=0
$$

and

$$
\lim _{t \rightarrow 0}|t|^{-2} g(t)=0 \text {. }
$$

For any radially symmetric sequence $\left\{u_{k}\right\}_{k}$ satisfying $\|\Delta u\|_{2}^{2} \leq 32 \pi^{2} K$, and weakly converging to $u$, we will verify that

$$
\lim _{k \rightarrow \infty} G\left(u_{k}\right)-G(u)=\lim _{k \rightarrow \infty} \int_{\mathbb{R}^{4}}\left(g\left(u_{k}\right)-g(u)\right) d x=0 .
$$


Note that $\left\{u_{k}\right\}_{k}$ is a radial sequence in $H^{2}\left(\mathbb{R}^{4}\right)$, then

$$
\begin{aligned}
\left|u_{k}(r)\right|^{2} & \leq \int_{r}^{+\infty} 2\left|u_{k}(s)\right|\left|u_{k}^{\prime}(s)\right| d s \\
& \leq c r^{-3} \int_{r}^{+\infty}\left|u_{k}(s) s^{3 / 2}\right|\left|u_{k}^{\prime}(s) s^{3 / 2}\right| d s \\
& \leq c r^{-3}\left(\int_{\mathbb{R}^{4}}\left|\nabla u_{k}\right|^{2} d x\right)^{1 / 2}\left(\int_{\mathbb{R}^{4}}\left|u_{k}\right|^{2} d x\right)^{1 / 2} .
\end{aligned}
$$

Hence $u_{k}(r) \rightarrow 0$ as $r \rightarrow \infty$ uniformly with respect to $k$. This together with (3.1) yields that for any $\varepsilon>0$, there exists $R>0$ such that

$$
\int_{\mathbb{R}^{4} \backslash B_{R}} g\left(u_{k}\right) d x \leq \varepsilon \int_{\mathbb{R}^{4}}\left|u_{k}\right|^{2} d x \leq c \varepsilon, \int_{\mathbb{R}^{4} \backslash B_{R}} g(u) d x \leq \varepsilon .
$$

On the other hand, through (3.2) we derive that for any $\varepsilon>0$, there exists $L>0$ independent of $k$ such that

$$
\int_{\left|u_{k}\right|>L} g\left(u_{k}\right) d x \leq c \varepsilon \int_{\left|u_{k}\right|>L} \frac{\exp \left(-\frac{1}{K}\left|u_{k}\right|^{2}\right)}{\left|u_{k}\right|^{2}} d x
$$

and

$$
\int_{|u|>L} g(u) d x \leq c \varepsilon \int_{|u|>L} \frac{\exp \left(-\frac{1}{K}|u|^{2}\right)}{|u|^{2}} d x .
$$

In view of (1.11), we derive that

$$
\int_{\left|u_{k}\right|>L} g\left(u_{k}\right) d x \leq c \varepsilon \int_{\mathbb{R}^{4}}\left|u_{k}\right|^{2} d x \leq c \varepsilon, \quad \int_{|u|>L} g(u) d x \leq \varepsilon .
$$

Combining (3.3) and (3.4), one can get

$$
\begin{aligned}
\lim _{k \rightarrow \infty}\left|G\left(u_{k}\right)-G(u)\right| \leq & \left(\int_{\mathbb{R}^{4} \backslash B_{R}}+\int_{B_{R}}\right)\left|g\left(u_{k}\right)-g(u)\right| d x \\
\leq & c \varepsilon+\lim _{k \rightarrow \infty}\left(\int_{\left|u_{k}\right|>L} g\left(u_{k}\right) d x+\int_{|u|>L} g(u) d x\right) \\
& +\lim _{k \rightarrow \infty}\left(\int_{\left|u_{k}\right| \leq L,|x| \leq R} g\left(u_{k}\right) d x-\int_{|u| \leq L,|x| \leq R} g(u) d x\right) \\
\leq & c \varepsilon+\lim _{k \rightarrow \infty}\left(\int_{\left|u_{k}\right| \leq L,|x| \leq R} g\left(u_{k}\right) d x-\int_{|u| \leq L,|x| \leq R} g(u) d x\right) \\
\leq & c \varepsilon,
\end{aligned}
$$

where we have used the Lebesgue dominated convergence theorem in the last step. Then the proof is finished. 
GROUND STATES OF BI-HARMONIC EQUATIONS WITH CRITICAL EXPONENTIAL GROWTH 13

\section{EXISTENCE OF NON-TRIVIAL SOLUTIONS FOR SEMILINEAR BI-HARMONIC EQUATIONS}

In this section, we consider the nontrivial solutions of semilinear bi-harmonic equation (2.1). We will employ the compactness result obtained in Theorem 2.1 and the principle of symmetric criticality to prove that equation (2.1) has a nontrivial radial solution under the assumption that the nonlinearity $f(t)$ satisfies mild conditions (i), (ii) and (1.2).

The natural functional associated to a variational approach to problem (2.1) is

$$
I_{\gamma}(u)=\frac{1}{2}\left(\|\Delta u\|_{2}^{2}+\gamma\|u\|_{2}^{2}\right)-\int_{\mathbb{R}^{4}} F(u) d x, \forall u \in H^{2}\left(\mathbb{R}^{4}\right) .
$$

Obviously, $I_{\gamma} \in C^{1}\left(H^{2}\left(\mathbb{R}^{4}\right), \mathbb{R}\right)$ with

$$
I_{\gamma}^{\prime}(u) v=\int_{\mathbb{R}^{4}}(\Delta u \Delta v+\gamma u v) d x-\int_{\mathbb{R}^{4}} f(u) v d x, \quad \forall u, v \in H^{2}\left(\mathbb{R}^{4}\right) .
$$

Our goal is to prove the existence of non-trivial solutions of the equation (2.1). According to the principle of symmetric criticality, we only need to verify that $u$ is a critical point restricted to the space $H_{r}^{2}\left(\mathbb{R}^{4}\right)$. Motivated by the Pohozaev identity for equation (2.1), we introduce the functional

$$
G_{\gamma}(u)=\gamma\|u\|_{2}^{2}-2 \int_{\mathbb{R}^{4}} F(u) d x
$$

and the constrained minimization problem

$$
\begin{aligned}
A_{\gamma} & =\inf \left\{\frac{1}{2}\|\Delta u\|_{2}^{2} \mid u \in H_{r}^{2}\left(\mathbb{R}^{4}\right), G_{\gamma}(u)=0\right\} \\
& =\inf \left\{I_{\gamma}(u) \mid u \in H_{r}^{2}\left(\mathbb{R}^{4}\right), G_{\gamma}(u)=0\right\} .
\end{aligned}
$$

Set $\mathcal{P}_{r}=\left\{u \in H_{r}^{2}\left(\mathbb{R}^{4}\right), G_{\gamma}(u)=0\right\}$. Apparently, $\mathcal{P}_{r}$ is not empty. In fact, let $u_{0} \in$ $H_{r}^{2}\left(\mathbb{R}^{4}\right)$ be compactly supported and define

$$
h(s):=G_{\gamma}\left(s u_{0}\right)=\gamma s^{2}\left\|u_{0}\right\|_{2}^{2}-2 \int_{\mathbb{R}^{4}} F\left(s u_{0}\right) d x, \forall s>0 .
$$

It follows from the fact

$$
\lim _{s \rightarrow 0^{+}} \frac{F(s)}{s^{2}}=0, \lim _{s \rightarrow+\infty} \frac{F(s)}{s^{2}}=\infty
$$

that $h(s)>0$ for $s>0$ small enough and $h(s)<0$ for $s>0$ sufficiently large. Therefore, there exists $s_{0}>0$ such that $h\left(s_{0} u_{0}\right)=0$. This gives $s_{0} u_{0} \in \mathcal{P}_{r}$.

Lemma 4.1. There exists a minimizing sequence $\left\{u_{k}\right\}_{k} \in \mathcal{P}_{r}$ satisfying $\left\|u_{k}\right\|_{2}=1$ for $A_{\gamma}$.

Proof. Assume that $\left\{u_{k}\right\}_{k}$ is a minimizing sequence for $A_{\gamma}$, that is, $u_{k} \in \mathcal{P}_{r}$ satisfying

$$
\lim _{k \rightarrow \infty} \frac{1}{2}\left\|\Delta u_{k}\right\|_{2}^{2}=A_{\gamma}
$$


Let $\tilde{v}_{k}=u_{k}\left(\left\|u_{k}\right\|_{2}^{1 / 2} x\right)$, simple computations lead to $\left\|\tilde{v}_{k}\right\|_{2}=1, \tilde{v}_{k} \in \mathcal{P}_{r}$ and $\left\|\Delta v_{k}\right\|_{2}=$ $\left\|\Delta \tilde{v}_{k}\right\|_{2}$. This accomplishes the proof of Lemma 4.1.

If the infimum $A_{\gamma}$ is attained, then the minimizer $u \in H_{r}^{2}\left(\mathbb{R}^{4}\right)$ under a suitable change of scale is a ground state solution of (2.1) constrained to the space $H_{r}^{2}\left(\mathbb{R}^{4}\right)$. In fact, if $u$ is a minimizer for $A_{\gamma}$, then there exists a Lagrange multiplier $\theta \in \mathbb{R}$ such that

$$
\Delta^{2} u+\gamma u-f(u)=\theta(2 \gamma u-2 f(u)) \text { in } \mathbb{R}^{4}
$$

namely,

$$
\Delta^{2} u=(2 \theta-1)(\gamma u-f(u)) \text { in } \mathbb{R}^{4} .
$$

Recalling that $u \in \mathcal{P}_{r}$, we have

$$
\begin{aligned}
\int_{\mathbb{R}^{4}}(\gamma u-f(u)) u d x & =\gamma\|u\|_{2}^{2}-2 \int_{\mathbb{R}^{4}} F(u) d x+\int_{\mathbb{R}^{4}}(2 F(u)-u f(u)) d x \\
& =-\int_{\mathbb{R}^{4}}(u f(u)-2 F(u)) d x<0,
\end{aligned}
$$

as a consequence of (i). Moreover,

$$
\int_{\mathbb{R}^{4}} \Delta^{2} u \cdot u d x=\int_{\mathbb{R}^{4}}|\Delta u|^{2} d x>0,
$$

hence $2 \theta-1<0$. Therefore

$$
\tilde{u}(x)=u\left(\frac{x}{(1-2 \theta)^{\frac{1}{2}}}\right) \text { for a.e. } x \in \mathbb{R}^{4}
$$

is a non-trivial solution of (2.1) constrained to the space $H_{r}^{2}\left(\mathbb{R}^{4}\right)$. According to the principle of symmetric criticality, then $u$ is a non-trivial solution of (2.1).

Now, we establish an relation between the attainability of $A_{\gamma}$ and the Adams' inequality with the exact growth (1.11). For this purpose, we introduce the Adams ratio

$$
C_{A}^{L}=\sup \left\{\frac{2}{\|u\|_{2}^{2}} \int_{\mathbb{R}^{4}} F(u) \mid u \in H_{r}^{2}\left(\mathbb{R}^{4}\right),\|\Delta u\|_{2}^{2} \leq L\right\} .
$$

The Adams threshold $R(F)$ is given by

$$
R(F)=\sup \left\{L>0 \mid C_{A}^{L}<+\infty\right\} .
$$

We denote by $C_{A}^{*}=C_{A}^{R(F)}$ the ratio at the threshold $R(F)$. By the growth condition (1.2) and (ii) of $f(s)$, we obtain

$$
\lim _{t \rightarrow+\infty} \frac{t^{2} F(t)}{\exp \left(\alpha t^{2}\right)}=\left\{\begin{array}{c}
0, \text { if } \alpha>\alpha_{0}, \\
+\infty, \text { if } \alpha<\alpha_{0}
\end{array}\right.
$$

and

$$
\lim _{t \rightarrow 0^{+}} \frac{F(t)}{t^{2}}=0 .
$$

Hence, thanks to Theorem [2.1, we derive $R(F)=32 \pi^{2} / \alpha_{0}$. 
Lemma 4.2. If $A_{\gamma}<R(F) / 2$, then $A_{\gamma}$ can be attained and $A_{\gamma}=I_{\gamma}(u)$, where $u \in H_{r}^{2}\left(\mathbb{R}^{4}\right)$ under a suitable change of scale is a nontrivial solution of equation (2.1) through the principle of symmetric criticality.

Proof. Let $\left\{u_{k}\right\}_{k}$ be a radial minimizing sequence for $A_{\gamma}$, that is $u_{k} \in \mathcal{P}_{r}$ satisfying

$$
\lim _{k \rightarrow \infty} \frac{1}{2}\left\|\Delta u_{k}\right\|_{2}^{2}=A_{\gamma} \text { and }\left\|u_{k}\right\|_{2}^{2}=1 .
$$

We also assume that $u_{k} \rightarrow u$ in $H^{2}\left(\mathbb{R}^{4}\right)$. We first prove that $A_{\gamma}>0$. We argue this by contradiction. We assume that $A_{\gamma}=0$, nemely $\lim _{k \rightarrow \infty}\left\|\Delta u_{k}\right\|_{2}^{2}=0$, which implies that $u=0$. Regarding

$$
\lim _{t \rightarrow+\infty}|t|^{2} \exp \left(-\alpha|t|^{2}\right) F(t)=0 \text { for any } \alpha>\alpha_{0}, \quad \lim _{t \rightarrow 0}|t|^{-2} F(t)=0,
$$

we derive that

$$
\lim _{k \rightarrow \infty} \int_{\mathbb{R}^{4}} F\left(u_{k}\right) d x=\int_{\mathbb{R}^{4}} F(u) d x
$$

through Theorem 2.2. On the other hand, since $u_{k} \in \mathcal{P}_{r}$ and $\left\|u_{k}\right\|_{2}^{2}=1$, then

$$
0<\gamma \lim _{k \rightarrow \infty}\left\|u_{k}\right\|_{2}^{2}=2 \lim _{k \rightarrow \infty} \int_{\mathbb{R}^{4}} F\left(u_{k}\right) d x=2 \int_{\mathbb{R}^{4}} F(u) d x
$$

which contradicts $u=0$. This proves that $A_{\gamma}>0$.

Now are in position to prove that if $A_{\gamma}<R(F) / 2$, then $A_{\gamma}$ could be attained. Under the assumption of Lemma 4.2, we have

$$
\lim _{k \rightarrow \infty}\left\|\Delta u_{k}\right\|_{2}^{2}=2 A_{\gamma}<R(F)=32 \pi^{2} / \alpha_{0} .
$$

Picking up $\frac{1}{K}>\alpha_{0}$ satisfying $\lim _{k \rightarrow \infty}\left\|\Delta u_{k}\right\|_{2}^{2} \leq 32 \pi^{2} K$, then we derive that

$$
\lim _{t \rightarrow+\infty}|t|^{2} \exp \left(-\frac{1}{K}|t|^{2}\right) F(t)=0, \lim _{t \rightarrow 0}|t|^{-2} F(t)=0 .
$$

It follows from Theorem 2.2 that

$$
\lim _{k \rightarrow \infty} \int_{\mathbb{R}^{4}} F\left(u_{k}\right) d x=\int_{\mathbb{R}^{4}} F(u) d x .
$$

Consequently,

$$
\gamma=\lim _{k \rightarrow \infty} \gamma\left\|u_{k}\right\|_{2}^{2}=2 \lim _{k \rightarrow \infty} \int_{\mathbb{R}^{4}} F\left(u_{k}\right) d x=2 \int_{\mathbb{R}^{4}} F(u) d x
$$

and

$$
\frac{1}{2}\|\Delta u\|_{2}^{2} \leq \lim _{k \rightarrow \infty} \frac{1}{2}\left\|\Delta u_{k}\right\|_{2}^{2}=A_{\gamma}
$$

In order to show $u$ is minimizer for $A_{\gamma}$, what left is to show that $G_{\gamma}(u)=0$. Set

$$
h(t)=G_{\gamma}(t u)=\gamma\|t u\|_{2}^{2}-\int_{\mathbb{R}^{4}} F(t u) d x .
$$


Obviously, in view of (4.3), we have

$$
\begin{aligned}
G_{\gamma}(u) & =\gamma\|u\|_{2}^{2}-2 \int_{\mathbb{R}^{4}} F(u) d x \\
& \leq \lim _{k \rightarrow \infty}\left(\gamma\left\|u_{k}\right\|_{2}^{2}-\int_{\mathbb{R}^{4}} F\left(u_{k}\right) d x\right)=\lim _{k \rightarrow \infty} G_{\gamma}\left(u_{k}\right)=0 .
\end{aligned}
$$

This implies $h(1) \leq 0$. From $\lim _{t \rightarrow 0^{+}} \frac{F(t)}{t^{2}}=0$, one can deduce that $h(t)>0$ for $t>0$ small enough. Consequently, there exists $s_{0} \in(0,1]$ such that $G_{\gamma}\left(s_{0} u\right)=0$. Then it follows that

$$
A_{\gamma} \leq \frac{1}{2}\left\|\Delta s_{0} u\right\|_{2}^{2}=\frac{1}{2} s_{0}^{2}\|\Delta u\|_{2}^{2} \leq s_{0}^{2} A_{\gamma}
$$

which proves that $s_{0}=1$ and $\frac{1}{2}\|\Delta u\|_{2}^{2}=A_{\gamma}$. Then we accomplish the proof of Lemma 4.2 .

Next, we show

Lemma 4.3. The constrained minimization problem $A_{\gamma}$ associated to the functional $I_{\gamma}$ satisfies

$$
A_{\gamma}<\frac{1}{2} R(F)
$$

if and only if

$$
\gamma<C_{A}^{*}
$$

Proof. We first prove that if $A_{\gamma}<R(F) / 2$, then $\gamma<C_{A}^{*}$. Obviously, if the $C_{A}^{*}=+\infty$, then $\gamma<C_{A}^{*}$ and the proof is complete. Therefore, without loss of generality, we may assume that $C_{A}^{*}<+\infty$. According to Lemma 4.2, we see that $A_{\gamma}$ could be achieved by a radial function $u \in \mathcal{P}_{r}$. Then according to the definition of the $A_{\gamma}$, we have $\|\Delta u\|_{2}^{2}<32 \pi^{2} / \alpha_{0}$ and $\gamma\|u\|_{2}^{2}=2 \int_{\mathbb{R}^{4}} F(u) d x$. Define

$$
g(s)=\frac{2}{s^{2}\|u\|_{2}^{2}} \int_{\mathbb{R}^{4}} F(s u) d x,
$$

then $g(1)=\gamma$. Since $F$ satisfies the condition (i), then it is easy to see that $g(s)$ is monotone increasing. If we set $v=\frac{R(F)^{1 / 2}}{\|\Delta u\|_{2}} u$, then $\|\Delta v\|_{2}^{2}=R(F)$ and

$$
C_{A}^{*} \geq \frac{2}{\|v\|_{2}^{2}} \int_{\mathbb{R}^{4}} F(v) d x=g\left(\frac{R(F)^{1 / 2}}{\|\Delta u\|_{2}}\right)>g(1)=\gamma .
$$

Next, it remains to verify that if $\gamma<C_{A}^{*}$, then $A_{\gamma}<R(F) / 2$. We distinguish between the case $C_{A}^{*}<+\infty$ and $C_{A}^{*}=+\infty$.

In the case $C_{A}^{*}<+\infty$, since $\gamma<C_{A}^{*}$, then $\gamma<C_{A}^{*}-\varepsilon_{0}$ for some $\varepsilon_{0}>0$. It follows from the definition of $C_{A}^{*}$ that there exists some $u_{0} \in H_{r}^{2}\left(\mathbb{R}^{4}\right)$ with $\left\|\Delta u_{0}\right\|_{2}^{2} \leq R(F)$ satisfying

$$
C_{A}^{*}-\varepsilon_{0}<\frac{2}{\left\|u_{0}\right\|_{2}^{2}} \int_{\mathbb{R}^{4}} F\left(u_{0}\right) d x .
$$


GROUND STATES OF BI-HARMONIC EQUATIONS WITH CRITICAL EXPONENTIAL GROWTH 17

Consequently,

$$
\gamma\left\|u_{0}\right\|_{2}^{2}<2 \int_{\mathbb{R}^{4}} F\left(u_{0}\right) d x
$$

namely $G_{\gamma}(u)<0$. Let $h(s)=G_{\gamma}\left(s u_{0}\right)$ for $s>0$. Since $h(1)<0$ and $h(s)>0$ for $s>0$ small enough, then there exists $s_{0} \in(0,1)$ satisfying $h\left(s_{0} u_{0}\right)=0$. Therefore, we have $s_{0} u_{0} \in \mathcal{P}_{r}$ and

$$
A_{\gamma} \leq \frac{1}{2}\left\|\Delta\left(s_{0} u_{0}\right)\right\|_{2}^{2}=\frac{1}{2} s_{0}^{2}\left\|\Delta u_{0}\right\|_{2}^{2}<\frac{1}{2} R(F) .
$$

In the case $C_{A}^{*}=+\infty$, for any $\gamma>0$, there exists $u_{0} \in H_{r}^{2}\left(\mathbb{R}^{4}\right)$ with $\left\|\Delta u_{0}\right\|_{2}^{2} \leq R(F)$ satisfying

$$
\gamma\left\|u_{0}\right\|_{2}^{2}<2 \int_{\mathbb{R}^{4}} F\left(u_{0}\right) d x .
$$

Hence we can repeat the same arguments as case $C_{A}^{*}<+\infty$ to get the conclusion.

\section{EXISTENCE OF GROUND STATE SOLUTIONS FOR BI-HARMONIC EQUATION With THE CONSTANT POTENTIAL}

In this section, we will employ the Pohozaev manifold and Fourier rearrangement arguments to study the ground-states of the following semilinear bi-harmonic equation.

$$
(-\Delta)^{2} u+\gamma u=\lambda u \exp \left(2|u|^{2}\right) \text { in } \mathbb{R}^{4},
$$

where $\lambda$ is strictly smaller than the first eigenvalue of operator $(-\Delta)^{2}+\gamma I$ in $\mathbb{R}^{4}$, namely

$$
\lambda<\inf _{u \in H^{2}\left(\mathbb{R}^{4}\right)} \frac{\|\Delta u\|_{2}^{2}+\gamma\|u\|_{2}^{2}}{\|u\|_{2}^{2}}=\gamma .
$$

The natural functional associated to a variational approach to problem (5.1) is

$$
I_{\lambda}(u)=\frac{1}{2}\left(\|\Delta u\|_{2}^{2}+\gamma\|u\|_{2}^{2}\right)-\frac{\lambda}{4} \int_{\mathbb{R}^{4}}\left(\exp \left(2 u^{2}\right)-1\right) d x, \forall u \in H^{2}\left(\mathbb{R}^{4}\right) .
$$

It is easy to obtain that $I_{\lambda} \in C^{1}\left(H^{2}\left(\mathbb{R}^{4}\right), \mathbb{R}\right)$ with

$$
I_{\lambda}^{\prime}(u) v=\int_{\mathbb{R}^{4}}(\Delta u \Delta v+\gamma u v) d x-\int_{\mathbb{R}^{4}} \lambda u \exp \left(2 u^{2}\right) v d x, \forall u, v \in H^{2}\left(\mathbb{R}^{4}\right) .
$$

We will prove that equation (5.1) has a radial ground-state solution for any $0<\lambda<\gamma$.

We recall that a solution $u$ of (5.1) is called a ground state if $I_{\lambda}(u)=m_{\lambda}$, where

$$
m_{\lambda}=\inf \left\{I_{\lambda}(u) \mid u \text { is a weak solution of (5.1) }\right\} \text {. }
$$

Similar to the proof of Theorem 2.3, we introduce the Pohozaev functional

$$
\left.G_{\lambda}(u)=c\|u\|_{2}^{2}-\frac{1}{2} \int_{\mathbb{R}^{4}} \lambda\left(\exp \left(2|u|^{2}\right)-1\right)\right) d x=(\gamma-\lambda)\|u\|_{2}^{2}-\int_{\mathbb{R}^{4}} g_{\lambda}(u) d x,
$$


where $g_{\lambda}(t)=\frac{\lambda}{2}\left(\exp \left(2 t^{2}\right)-1-2 t^{2}\right)$, and the constrained minimization problem

$$
\begin{aligned}
A_{\lambda} & =\inf \left\{\frac{1}{2}\|\Delta u\|_{2}^{2} \mid u \in H^{2}\left(\mathbb{R}^{4}\right), G_{\lambda}(u)=0\right\} \\
& =\inf \left\{I_{\lambda}(u) \mid u \in H^{2}\left(\mathbb{R}^{4}\right), G_{\lambda}(u)=0\right\} \leq m_{\lambda},
\end{aligned}
$$

Set $\mathcal{P}=\left\{u \in H^{2}\left(\mathbb{R}^{4}\right), G_{\lambda}(u)=0\right\}$, obviously $\mathcal{P}$ is not empty. Next, we will adapt the Fourier rearrangement method to show that there exists a radially minimizing sequence for $A_{\lambda}$. Such a Fourier rearrangement argument has also been used recently by Chen, $\mathrm{Lu}$ and Zhang in [15] to establish the existence of extremals for the subcritical Adams inequalities on the entire space.

Lemma 5.1. There exists a radially minimizing sequence $\left\{u_{k}\right\}_{k}$ satisfying $\left\|u_{k}\right\|_{2}^{2}=1$ for $A_{\lambda}$.

Proof. Assume that $\left\{u_{k}\right\}_{k}$ is a minimizing sequence for $A_{\lambda}$, that is $u_{k} \in \mathcal{P}$ satisfying

$$
\lim _{k \rightarrow \infty} \frac{1}{2}\left\|\Delta u_{k}\right\|_{2}^{2}=A_{\lambda}
$$

Denote by $w_{k}=\mathcal{F}^{-1}\left\{\left(\mathcal{F}\left(u_{k}\right)\right)^{*}\right\}$ the Fourier rearrangement of $u_{k}$, where $\mathcal{F}$ is the Fourier transform on $\mathbb{R}^{4}$ (with its inverse $\mathcal{F}^{-1}$ ) and $f^{*}$ stands for the Schwarz symmetrization of $f$. Using the property of the Fourier rearrangement from [30], one can derive that

$$
\begin{aligned}
\left\|\Delta w_{k}\right\|_{2} & \leq\left\|\Delta u_{k}\right\|_{2},\left\|w_{k}\right\|_{2}^{2}=\left\|u_{k}\right\|_{2}^{2}, \\
\int_{\mathbb{R}^{4}}\left(\exp \left(2 w_{k}^{2}\right)-1\right) d x & \geq \int_{\mathbb{R}^{4}}\left(\exp \left(2 u_{k}^{2}\right)-1\right) d x .
\end{aligned}
$$

Then it follows that

$$
(\gamma-\lambda)\left\|w_{k}\right\|_{2}^{2}=(\gamma-\lambda)\left\|u_{k}\right\|_{2}^{2}=\int_{\mathbb{R}^{4}} g_{\lambda}\left(u_{k}\right) d x \leq \int_{\mathbb{R}^{4}} g_{\lambda}\left(w_{k}\right) .
$$

Hence if we set

$$
\eta(t)=(\gamma-\lambda)\left\|t w_{k}\right\|_{2}^{2}-\int_{\mathbb{R}^{4}} g_{\lambda}\left(t w_{k}\right),
$$

then $\eta(1) \leq 0$. On the other hand, one can easily see $\eta(t)>0$ for $t>0$ sufficiently small. Therefore, there exists $t_{k} \in(0,1]$ such that $\eta\left(t_{k}\right)=0$, that is $t_{k} w_{k} \in \mathcal{P}$. We obtain

$$
m_{\lambda} \leq I_{\lambda}\left(t_{k} w_{k}\right)=\frac{1}{2}\left\|\Delta\left(t_{k} w_{k}\right)\right\|_{2}^{2} \leq \frac{1}{2} t_{k}^{2}\left\|\Delta u_{k}\right\|_{2}^{2} \leq I_{\lambda}\left(u_{k}\right) .
$$

This implies that $\left\{v_{k}\right\}:=\left\{t_{k} w_{k}\right\}_{k}$ is a radial minimizing sequence for $m_{\lambda}$. Let $\tilde{v}_{k}=$ $w_{k}\left(\left\|v_{k}\right\|_{2}^{1 / 2} x\right)$, it is easy to check that $\tilde{v}_{k}$ is a minimizing sequence for $A_{\lambda}$ with $\left\|\tilde{v}_{k}\right\|_{2}=1$. This accomplishes the proof of Lemma 5.1.

Repeating the argument for (4.2), we can show that if the infimum $A_{\lambda}$ is attained, then the minimizer $u \in H_{r}^{2}\left(\mathbb{R}^{4}\right)$ under a suitable change of scale is a ground state solution of (5.1). 
Lemma 5.2. If $A_{\lambda}<8 \pi^{2}$, then $A_{\lambda}$ could be attained and $A_{\lambda}=I_{\lambda}(u)$, where $u \in H_{r}^{2}\left(\mathbb{R}^{4}\right)$ under a suitable change of scale is a ground-state solution of equation (5.1).

Proof. Let $\left\{u_{k}\right\}_{k}$ is a radial minimizing sequence for $A_{\lambda}$, that is $u_{k} \in \mathcal{P}$ satisfying

$$
\lim _{k \rightarrow \infty} \frac{1}{2}\left\|\Delta u_{k}\right\|_{2}^{2}=A_{\lambda} \text { and }\left\|u_{k}\right\|_{2}^{2}=1
$$

We also assume that $u_{k} \rightarrow u$ in $H^{2}\left(\mathbb{R}^{4}\right)$. We first prove that $A_{\lambda}>0$. By way of contradiction, we assume that $A_{\lambda}=0$, namely $\lim _{k \rightarrow \infty}\left\|\Delta u_{k}\right\|_{2}^{2}=0$. This implies $u=0$. Since

$$
\lim _{t \rightarrow+\infty}|t|^{2} \exp \left(-\alpha|t|^{2}\right) g_{\lambda}(t)=0 \text { for any } \alpha>2, \quad \lim _{t \rightarrow 0}|t|^{-2} g_{\lambda}(t)=0
$$

It follows from Theorem 2.2 that

$$
\lim _{k \rightarrow \infty} \int_{\mathbb{R}^{4}} g_{\lambda}\left(u_{k}\right) d x=\int_{\mathbb{R}^{4}} g_{\lambda}(u) d x .
$$

On the other hand, since $u_{k} \in \mathcal{P}$ with $\left\|u_{k}\right\|_{2}^{2}=1$, we have

$$
0<(\gamma-\lambda) \leq \lim _{k \rightarrow \infty}(\gamma-\lambda)\left\|u_{k}\right\|_{2}^{2}=\lim _{k \rightarrow \infty} \int_{\mathbb{R}^{4}} g_{\lambda}\left(u_{k}\right) d x=\int_{\mathbb{R}^{4}} g_{\lambda}(u) d x,
$$

which contradicts $u=0$. This proves that $A_{\lambda}>0$.

Now we are in position to prove that if $A_{\lambda}<8 \pi^{2}$, then $A_{\lambda}$ could be attained. Under the assumption of Lemma 5.2, we derive that $\lim _{k \rightarrow \infty}\left\|\Delta u_{k}\right\|_{2}^{2}=2 A_{\lambda}<16 \pi^{2}$. Setting $K=A_{\lambda} / 16 \pi^{2}$, observing that

$$
\lim _{t \rightarrow+\infty}|t|^{2} \exp \left(-\frac{1}{K}|t|^{2}\right) g_{\lambda}(t)=0 \text { and } \lim _{t \rightarrow 0}|t|^{-2} g_{\lambda}(t)=0,
$$

one can employ the compactness result obtained in Theorem 2.2 to derive that

$$
\lim _{k \rightarrow \infty} \int_{\mathbb{R}^{4}} g_{\lambda}\left(u_{k}\right) d x=\int_{\mathbb{R}^{4}} g_{\lambda}(u) d x .
$$

Consequently, we get

$$
(\gamma-\lambda)=\lim _{k \rightarrow \infty}(\gamma-\lambda)\left\|u_{k}\right\|_{2}^{2}=\lim _{k \rightarrow \infty} \int_{\mathbb{R}^{4}} g_{\lambda}\left(u_{k}\right) d x=\int_{\mathbb{R}^{4}} g_{\lambda}(u) d x
$$

and

$$
\frac{1}{2}\|\Delta u\|_{2}^{2} \leq \lim _{k \rightarrow \infty} \frac{1}{2}\left\|\Delta u_{k}\right\|_{2}^{2}=A_{\lambda}
$$

In order to show $u$ is minimizer for $A_{\lambda}$, we only need to verify that $G_{\lambda}(u)=0$. Set

$$
h(t)=G_{\lambda}(t u)=(\gamma-\lambda)\|t u\|_{2}^{2}-\int_{\mathbb{R}^{4}} g_{\lambda}(t u) d x .
$$


Since

$$
\begin{aligned}
G_{\lambda}(u) & =(\gamma-\lambda)\|u\|_{2}^{2}-\int_{\mathbb{R}^{4}} g_{\lambda}(u) d x \\
& \leq \lim _{k \rightarrow \infty}(\gamma-\lambda)\left\|u_{k}\right\|_{2}^{2}-\int_{\mathbb{R}^{4}} g_{\lambda}\left(u_{k}\right) d x=\lim _{k \rightarrow \infty} G_{\lambda}\left(u_{k}\right)=0,
\end{aligned}
$$

then $h(1) \leq 0$. In view of $\lim _{t \rightarrow 0^{+}} \frac{g_{\lambda}(t)}{t^{2}}=0$, one can deduce that $h(t)>0$ for $t>0$ small enough. Consequently, there exists $s_{0} \in(0,1]$ such that $G_{\lambda}\left(s_{0} u\right)=0$. By (5.2), we obtain

$$
A_{\lambda} \leq \frac{1}{2}\left\|\Delta s_{0} u\right\|_{2}^{2}=\frac{1}{2} s_{0}^{2}\|\Delta u\|_{2}^{2} \leq s_{0}^{2} A_{\lambda}
$$

which leads to $s_{0}=1$ and $\frac{1}{2}\|\Delta u\|_{2}^{2}=A_{\lambda}$. Then we accomplish the proof of Lemma 5.2 .

Next, we prove the

Lemma 5.3. The constrained minimization problem $A_{\lambda}$ is actually strictly smaller than $8 \pi^{2}$.

Proof. Note that the Adams ratio for $g_{\lambda}(u)$ is $+\infty$, hence there exists $u_{0} \in H^{2}\left(\mathbb{R}^{4}\right)$ such that

$$
(\gamma-\lambda) \leq \frac{1}{\left\|u_{0}\right\|_{2}^{2}} \int_{\mathbb{R}^{4}} g_{\lambda}\left(u_{0}\right) d x, \quad\left\|\Delta u_{0}\right\|_{2}^{2} \leq 16 \pi^{2},
$$

thus, we have $G_{\lambda}\left(u_{0}\right)=(\gamma-\lambda)\left\|u_{0}\right\|_{2}^{2}-\int_{\mathbb{R}^{4}} g_{\lambda}\left(u_{0}\right) d x<0$. Then there exists $s_{0} \in(0,1)$ such that $s_{0} u_{0} \in \mathcal{P}$, which yields that

$$
A_{\lambda} \leq \frac{1}{2}\left\|\Delta\left(s_{0} u_{0}\right)\right\|_{2}^{2}=\frac{1}{2} s_{0}^{2}\left\|\Delta u_{0}\right\|_{2}^{2} \leq 8 \pi^{2} s_{0}^{2}<8 \pi^{2} .
$$

Then the lemma is proved.

\section{EXISTENCE OF GROUND STATE SOLUTIONS FOR BI-HARMONIC EQUATION WITH} THE RABINOWITZ TYPE POTENTIAL

In this section, we are concerned with the ground states of the following quasilinear bi-harmonic equation with the Rabinowitz type potential

$$
(-\Delta)^{2} u+V(x) u=\lambda \exp \left(2 u^{2}\right) u,
$$

where $\lambda$ and $V(x)$ satisfy

$$
0<\lambda<V_{0}=\inf _{x \in \mathbb{R}^{4}} V(x)<\lim _{|x| \rightarrow \infty} V(x)=\gamma .
$$

The associated functional and Nehari Manifold are

$$
I_{V}(u)=\frac{1}{2} \int_{\mathbb{R}^{4}}\left(|\Delta u|^{2}+V(x)|u|^{2}\right) d x-\frac{\lambda}{4} \int_{\mathbb{R}^{4}}\left(\exp \left(2 u^{2}\right)-1\right) d x
$$

and 


$$
\mathcal{N}_{V}=\left\{u \in H^{2}\left(\mathbb{R}^{4}\right) \mid u \neq 0, N_{V}(u)=0\right\}
$$

respectively, where

$$
N_{V}(u)=\int_{\mathbb{R}^{4}}\left(|\Delta u|^{2}+V(x)|u|^{2}\right) d x-\lambda \int_{\mathbb{R}^{4}} \exp \left(2 u^{2}\right) u^{2} d x .
$$

In order to study the equation (6.1), we introduce the following limiting equation

$$
(-\Delta)^{2} u+\gamma u=\lambda \exp \left(2 u^{2}\right) u \text {. }
$$

The corresponding functional and Nehari Manifold associated with (6.2) is

$$
I_{\infty}(u)=\frac{1}{2} \int_{\mathbb{R}^{4}}\left(|\Delta u|^{2}+\gamma|u|^{2}\right) d x-\frac{\lambda}{4} \int_{\mathbb{R}^{4}}\left(\exp \left(2 u^{2}\right)-1\right) d x
$$

and

$$
\mathcal{N}_{\infty}=\left\{u \in H^{2}\left(\mathbb{R}^{4}\right) \mid u \neq 0, N_{\infty}(u)=0\right\}
$$

where

$$
N_{\infty}(u)=\int_{\mathbb{R}^{4}}\left(|\Delta u|^{2}+\gamma|u|^{2}\right) d x-\lambda \int_{\mathbb{R}^{4}} \exp \left(2 u^{2}\right) u^{2} d x .
$$

One can easily verify that if $u \in \mathcal{N}_{V}$, then

$$
I_{V}(u)=\frac{\lambda}{4} \int_{\mathbb{R}^{4}}\left(\exp \left(2 u^{2}\right) 2 u^{2}-\left(\exp \left(2 u^{2}\right)-1\right)\right) d x
$$

and if $u \in \mathcal{N}_{\infty}$, then

$$
I_{\infty}(u)=\frac{\lambda}{4} \int_{\mathbb{R}^{4}}\left(\exp \left(2 u^{2}\right) 2 u^{2}-\left(\exp \left(2 u^{2}\right)-1\right)\right) d x .
$$

Lemma 6.1. For any $u \in H^{2}\left(\mathbb{R}^{4}\right)$, there exist unique $t_{u}$ and $\tilde{t}_{u}$ such that $t_{u} u \in \mathcal{N}_{q}$ and $\tilde{t}_{u} u \in \mathcal{N}_{\infty}$.

Proof. For any $u \in H^{2}\left(\mathbb{R}^{4}\right)$, we have

$$
N_{V}(t u)=t^{2} \int_{\mathbb{R}^{4}}\left(|\Delta u|^{2}+(V(x)-\lambda)|u|^{2}\right) d x-\lambda \int_{\mathbb{R}^{4}}\left(\exp \left(2 t^{2} u^{2}\right)-1\right) t^{2} u^{2} d x .
$$

Since

$$
\lim _{t \rightarrow 0} \frac{\left(\exp \left(2 t^{2} u^{2}\right)-1\right) t^{2} u^{2}}{t^{2}}=0 \text { and } \lim _{t \rightarrow \infty} \frac{\left(\exp \left(2 t^{2} u^{2}\right)-1\right) t^{2} u^{2}}{t^{2}}=+\infty,
$$

then $N_{V}(t u)>0$ for $s>0$ small enough and $N_{V}(t u)<0$ for $t$ sufficient large. With the help of the monotonicity of $\exp \left(2 t^{2} u^{2}\right)$, there exists a unique $t_{u}>0$ such that $t_{u} u \in \mathcal{N}_{V}$. The proof for $\mathcal{N}_{\infty}$ is similar. 
Set

$$
m_{\infty}=\inf \left\{I_{\infty}(u), u \in \mathcal{N}_{\infty}\right\} \text { and } m_{V}=\inf \left\{I_{V}(u), u \in \mathcal{N}_{V}\right\} .
$$

From Corollary 2.5, we know that $m_{\infty}$ is attained by some $w \in \mathcal{N}_{\infty}$.

Lemma 6.2. There holds

$$
0<m_{V}<m_{\infty}
$$

Proof. We first claim that for any $u \in m_{V}$, there exists some bounded $\tilde{t}_{u}>1$ such that $\tilde{t}_{u} u \in \mathcal{N}_{\infty}$. Indeed, for any $u \in \mathcal{N}_{V}$, by the assumption of $V(x)$, we have

$$
\begin{aligned}
\lambda \int_{\mathbb{R}^{4}} \exp \left(2 u^{2}\right) u^{2} d x & =\int_{\mathbb{R}^{4}}\left(|\Delta u|^{2}+V(x)|u|^{2}\right) d x \\
& <\int_{\mathbb{R}^{4}}\left(|\Delta u|^{2}+\gamma|u|^{2}\right) d x=\lambda \int_{\mathbb{R}^{4}} \exp \left(2 \tilde{t}_{u}^{2} u^{2}\right) u^{2} d x,
\end{aligned}
$$

which implies $\tilde{t}_{u}>1$. Now, we prove the boundedness of $\tilde{t}_{u}$. By the assumption of $V(x)$, there exists some constant $c>0$ such that

$$
\begin{aligned}
c \lambda \int_{\mathbb{R}^{4}} \exp \left(2 u^{2}\right) u^{2} d x & =c \int_{\mathbb{R}^{4}}\left(|\Delta u|^{2}+V(x)|u|^{2}\right) d x \\
& \geq \int_{\mathbb{R}^{4}}\left(|\Delta u|^{2}+(\gamma-\lambda)|u|^{2}\right) d x \\
& =\lambda \int_{\mathbb{R}^{4}}\left(\exp \left(2 \tilde{t}_{u}^{2} u^{2}\right)-1\right) u^{2} d x .
\end{aligned}
$$

Since $\lambda \int_{\mathbb{R}^{4}}\left(\exp \left(2 u^{2}\right)-1\right) u^{2} d x=\int_{\mathbb{R}^{4}}\left(|\Delta u|^{2}+(V(x)-\lambda)|u|^{2}\right) d x$, we derive that

$$
\begin{aligned}
\lambda \int_{\mathbb{R}^{4}}\left(\exp \left(2 \tilde{t}_{u}^{2} u^{2}\right)-1\right) u^{2} d x & \geq \lambda \tilde{t}_{u}^{2} \int_{\mathbb{R}^{4}}\left(\exp \left(2 u^{2}\right)-1\right) u^{2} d x \\
& =\tilde{t}_{u}^{2} \int_{\mathbb{R}^{4}}\left(|\Delta u|^{2}+(V(x)-\lambda)|u|^{2}\right) d x
\end{aligned}
$$

Combining (6.4) and (6.5), we conclude that

$$
\begin{aligned}
c \int_{\mathbb{R}^{4}}\left(|\Delta u|^{2}+V(x)|u|^{2}\right) d x & =c \lambda \int_{\mathbb{R}^{4}} \exp \left(2 u^{2}\right) u^{2} d x \\
& \geq \lambda \int_{\mathbb{R}^{4}}\left(\exp \left(2 \tilde{t}_{u}^{2} u^{2}\right)-1\right) u^{2} d x \\
& \geq \tilde{t}_{u}^{2} \int_{\mathbb{R}^{4}}\left(|\Delta u|^{2}+(V(x)-\lambda)|u|^{2}\right) d x .
\end{aligned}
$$

Therefore, $\tilde{t}_{u}$ must be bounded.

To show that $m_{V}<m_{\infty}$, it is enough to build a sequence $\left(u_{k}\right)_{k}$ satisfying $u_{k} \in \mathcal{N}_{V}$ such that $\lim _{k \rightarrow \infty} I_{V}\left(u_{k}\right) \leq m_{\infty}$. Consider $\left(y_{k}\right)_{k}$ with $y_{k} \in \mathbb{R}^{4}$ and $\left|y_{k}\right| \rightarrow \infty$. We define 
GROUND STATES OF BI-HARMONIC EQUATIONS WITH CRITICAL EXPONENTIAL GROWTH 23 $u_{k}=t_{k} w_{y_{k}}$, where $w_{y_{k}}=w\left(\cdot-y_{k}\right)$ and $t_{k}=t_{w_{y_{k}}}$ satisfying $u_{k}=t_{k} w_{y_{k}} \in \mathcal{N}_{V}$. Then we have

$$
\int_{\mathbb{R}^{4}}\left(|\Delta w|^{2}+\gamma|w|^{2}\right) d x=\lambda \int_{\mathbb{R}^{4}} \exp \left(2 w^{2}\right) w^{2} d x
$$

and

$$
\begin{aligned}
\lambda \int_{\mathbb{R}^{4}} \exp \left(2 t_{k}^{2} w^{2}\right) w^{2} d x & =\int_{\mathbb{R}^{4}}\left(\left|\Delta w_{y_{k}}\right|^{2}+V(x)\left|w_{y_{k}}\right|^{2}\right) d x \\
& =\int_{\mathbb{R}^{4}}\left(|\Delta w|^{2}+V\left(x+y_{k}\right)|w|^{2}\right) d x \\
& \rightarrow \int_{\mathbb{R}^{4}}\left(|\Delta w|^{2}+\gamma|w|^{2}\right) d x \\
& =\lambda \int_{\mathbb{R}^{4}} \exp \left(2 w^{2}\right) w^{2} d x
\end{aligned}
$$

This gives $t_{k} \rightarrow 1$ as $k \rightarrow \infty$. Observe that

$$
\begin{aligned}
& c\left(\exp \left(2 u^{2}\right) 2 u^{2}-2 u^{2}\right) \leq \exp \left(2 u^{2}\right) 2 u^{2}-\exp \left(2 u^{2}\right)+1 \\
& =\frac{\left(2 u^{2}\right)^{2}}{2}+\left(\frac{1}{2}-\frac{1}{3 !}\right)\left(2 u^{2}\right)^{3}+\left(\frac{1}{3 !}-\frac{1}{4 !}\right)\left(2 u^{2}\right)^{4}+\ldots \\
& +\left(\frac{1}{(n-1) !}-\frac{1}{n !}\right)\left(2 u^{2}\right)^{n}+\ldots \leq \exp \left(2 u^{2}\right) 2 u^{2}-2 u^{2}
\end{aligned}
$$

then

$$
\begin{aligned}
\lim _{k \rightarrow \infty} I_{V}\left(u_{k}\right) & =\lim _{k \rightarrow \infty} \frac{\lambda}{4} \int_{\mathbb{R}^{4}}\left(\exp \left(2 t_{k}^{2} w_{y_{k}}^{2}\right) 2 t_{k}^{2} w_{y_{k}}^{2}-\left(\exp \left(2 t_{k}^{2} w_{y_{k}}^{2}\right)-1\right)\right) d x \\
& =\lim _{k \rightarrow \infty} \frac{\lambda}{4} \int_{\mathbb{R}^{4}}\left(\exp \left(2 t_{k}^{2} w^{2}\right) 2 t_{k}^{2} w^{2}-\left(\exp \left(2 t_{k}^{2} w^{2}\right)-1\right)\right) d x \\
& \leq \frac{\lambda}{4} \int_{\mathbb{R}^{4}}\left(\exp \left(2 w^{2}\right) 2 w^{2}-\left(\exp \left(2 w^{2}\right)-1\right)\right) d x=m_{\infty} .
\end{aligned}
$$

Therefore, $m_{V}<m_{\infty}$ thanks to $t_{k}<1$.

Next, we show $m_{V}>0$. We prove this by contradiction. Assume that there exists some sequence $u_{k} \in \mathcal{N}_{V}$ such that $I_{V}\left(u_{k}\right) \rightarrow 0$, that is,

$$
\frac{\lambda}{4} \int_{\mathbb{R}^{4}}\left(\exp \left(2 u_{k}^{2}\right) 2 u_{k}^{2}-\exp \left(2 u_{k}^{2}\right)-1\right) d x \rightarrow 0 .
$$

This together with (6.6) and $N_{V}\left(u_{k}\right)=0$ implies that

$$
\int_{\mathbb{R}^{4}} \exp \left(2 u_{k}^{2}\right) 2 u_{k}^{2} d x \rightarrow 0 \text { and } \int_{\mathbb{R}^{4}}\left|\Delta u_{k}\right|^{2} d x \rightarrow 0 .
$$


Pick $\tilde{t}_{u_{k}}>0$ such that $\tilde{t}_{u_{k}} u_{k} \in \mathcal{N}_{\infty}$. Since $\tilde{t}_{u_{k}}$ is bounded, then $\int_{\mathbb{R}^{4}}\left|\Delta \tilde{t}_{u_{k}} u_{k}\right|^{2} d x \rightarrow 0$. Hence, it follows that

$$
\begin{aligned}
I_{\infty}\left(\tilde{t}_{u_{k}} u_{k}\right) & =\int_{\mathbb{R}^{4}}\left(\exp \left(2 \tilde{t}_{u_{k}}^{2} u_{k}^{2}\right) 2 \tilde{t}_{u_{k}}^{2} u_{k}^{2}-\left(\exp \left(2 \tilde{t}_{u_{k}}^{2} u_{k}^{2}\right)-1\right)\right) d x \\
& \leq c\left(\int_{\mathbb{R}^{4}}\left(\exp \left(2 \tilde{t}_{u_{k}}^{2} u_{k}^{2}\right)-1\right) 2 \tilde{t}_{u_{k}}^{2} u_{k}^{2} d x+\tilde{t}_{u_{k}}^{2} \int_{\mathbb{R}^{4}}\left|u_{k}\right|^{2} d x\right) \\
& \leq c \tilde{t}_{u_{k}}^{2} \int_{\mathbb{R}^{4}}\left(\left|\Delta u_{k}\right|^{2}+\left|u_{k}\right|^{2}\right) d x+\tilde{t}_{u_{k}}^{2} \int_{\mathbb{R}^{4}}\left|u_{k}\right|^{2} d x \rightarrow 0,
\end{aligned}
$$

where we have used the fact that

$$
\int_{\mathbb{R}^{4}}\left(\exp \left(2 u_{k}^{2}\right)-1\right) u_{k}^{2} d x \leq c \int_{\mathbb{R}^{4}}\left(\left|\Delta u_{k}\right|^{2}+\left|u_{k}\right|^{2}\right) d x
$$

provided that $\int_{\mathbb{R}^{4}}\left(\left|\Delta u_{k}\right|^{2}+\left|u_{k}\right|^{2}\right) d x$ is small enough. Next, we prove this fact. Indeed, direct computation yields

$$
\begin{aligned}
\int_{\mathbb{R}^{4}}\left(\exp \left(2 u_{k}^{2}\right)-1\right) u_{k}^{2} d x & \leq\left(\int_{\mathbb{R}^{4}}\left(\exp \left(2 u_{k}^{2}\right)-1\right)^{p} d x\right)^{1 / p}\left(\int_{\mathbb{R}^{4}} u_{k}^{2 p^{\prime}} d x\right)^{1 / p^{\prime}} \\
& \leq c\left(\int_{\mathbb{R}^{4}}\left(\exp \left(2 p u_{k}^{2}\right)-1\right) d x\right)^{1 / p}\left(\int_{\mathbb{R}^{4}} u_{k}^{2 p^{\prime}} d x\right)^{1 / p^{\prime}}
\end{aligned}
$$

where $p$ and $p^{\prime}$ satisfy $\frac{1}{p}+\frac{1}{p^{\prime}}=1$. Since $\int_{\mathbb{R}^{4}}\left(\left|\Delta u_{k}\right|^{2}+\left|u_{k}\right|^{2}\right) d x$ is small, then by the Adams' inequality (1.9) and the Sobolev inequality, we get

$$
\int_{\mathbb{R}^{4}}\left(\exp \left(2 u_{k}^{2}\right)-1\right) u_{k}^{2} d x \leq c \int_{\mathbb{R}^{4}}\left(\left|\Delta u_{k}\right|^{2}+\left|u_{k}\right|^{2}\right) d x .
$$

On the other hand, we have

$$
I_{\infty}\left(\tilde{t}_{u_{k}} u_{k}\right)=\frac{\lambda}{4} \int_{\mathbb{R}^{4}}\left(\exp \left(2 \tilde{t}_{u_{k}}^{2} u_{k}^{2}\right) 2 \tilde{t}_{u_{k}}^{2} u_{k}^{2}-\left(\exp \left(2 \tilde{t}_{u_{k}}^{2} u_{k}^{2}\right)-1\right)\right) d x \geq m_{\infty} .
$$

This is a contradiction. Therefore, $m_{V}>0$.

Lemma 6.3. The minimizing sequence $\left\{u_{k}\right\}_{k} \subset \mathcal{N}_{V}$ is bounded in $H^{2}\left(\mathbb{R}^{4}\right)$.

Proof. From (6.6), we know

$$
\begin{aligned}
\int_{\mathbb{R}^{4}}\left(\left|\Delta u_{k}\right|^{2}+(V(x)-\lambda)\left|u_{k}\right|^{2}\right) d x & =\frac{\lambda}{2} \int_{\mathbb{R}^{4}}\left(\exp \left(2 u_{k}^{2}\right)-1\right) 2 u_{k}^{2} d x \\
& \leq \frac{c \lambda}{2}\left(\int_{\mathbb{R}^{4}}\left(\exp \left(2 u_{k}^{2}\right) 2 u_{k}^{2}-\left(\exp \left(2 u_{k}^{2}\right)-1\right)\right) d x\right) \\
& \rightarrow 2 c m_{V} .
\end{aligned}
$$

Then the proof is finished from the assumption of $V(x)$.

We now consider a minimizing sequence $\left\{u_{k}\right\}_{k} \subset \mathcal{N}_{V}$. Since the sequence is bounded in $H^{2}\left(\mathbb{R}^{4}\right)$, then up to a subsequence, there exists $u \in H^{2}\left(\mathbb{R}^{4}\right)$, such that 
- $u_{k} \rightarrow u$ weakly in $H^{2}\left(\mathbb{R}^{4}\right)$ and in $L^{p}\left(\mathbb{R}^{4}\right)$, for any $p>1$,

- $u_{k} \rightarrow u$ in $L_{l o c}^{p}\left(\mathbb{R}^{4}\right)$,

- $u_{k} \rightarrow u$, a.e.

By extracting a subsequence, if necessary, we define $\beta, l \geq 0$ as

$$
\beta=\lim _{k} \int_{\mathbb{R}^{4}} \exp \left(2 u_{k}^{2}\right) u_{k}^{2} d x \text { and } l=\int_{\mathbb{R}^{4}} \exp \left(2 u^{2}\right) u^{2} d x .
$$

By the weak convergence, it is obvious that $l \in[0, \beta]$.

Lemma 6.4. There results $\beta>0$.

Proof. We argue this by contradiction. Assume that $\beta=0$. Since

$$
\int_{\mathbb{R}^{4}}\left(\exp \left(2 u_{k}^{2}\right)\right) 2 u_{k}^{2} d x>c \int_{\mathbb{R}^{4}}\left(\exp \left(2 u_{k}^{2}\right)-1\right) d x
$$

then $\int_{\mathbb{R}^{4}}\left(\exp \left(2 u_{k}^{2}\right)-1\right) d x \rightarrow 0$. Thus it follows that

$$
I_{V}\left(u_{k}\right)=\frac{\lambda}{4} \int_{\mathbb{R}^{4}}\left(\exp \left(2 u_{k}^{2}\right) 2 u_{k}^{2}-\left(\exp \left(2 u_{k}^{2}\right)-1\right)\right) d x \rightarrow 0,
$$

which contradicts (6.3).

Lemma 6.5. If $l=\beta$, then $u \in \mathcal{N}_{V}$ and $I_{V}(u)=m_{V}$.

Proof. If $l=\beta$, then $\int_{\mathbb{R}^{4}} \exp \left(2 u_{k}^{2}\right) u_{k}^{2} d x \rightarrow \int_{\mathbb{R}^{4}} \exp \left(2 u^{2}\right) u^{2} d x$ as $k \rightarrow+\infty$. Then one can get

$$
\begin{aligned}
\int_{\mathbb{R}^{4}}\left(|\Delta u|^{2}+V(x)|u|^{2}\right) d x & \leq \lim _{k \rightarrow \infty} \int_{\mathbb{R}^{4}}\left(\left|\Delta u_{k}\right|^{2}+V(x)\left|u_{k}\right|^{2}\right) d x \\
& =\lim _{k \rightarrow \infty} \lambda \int_{\mathbb{R}^{4}} \exp \left(2 u_{k}^{2}\right) u_{k}^{2} d x \\
& =\lim _{k \rightarrow \infty} \lambda \int_{\mathbb{R}^{4}} \exp \left(2 u^{2}\right) u^{2} d x .
\end{aligned}
$$

If the above equality holds, then $u \in \mathcal{N}_{V}$, and the lemma is proved. Therefore, it remains to show that the case

$$
\int_{\mathbb{R}^{4}}\left(|\Delta u|^{2}+V(x)|u|^{2}\right) d x<\lambda \int_{\mathbb{R}^{4}} \exp \left(2 u^{2}\right) u^{2} d x
$$

cannot occur. In fact, if (6.7) hold, we can take some $t \in(0,1)$ such that $t u \in \mathcal{N}_{V}$. Then we have

$$
\begin{aligned}
m_{\lambda} & \leq \frac{\lambda}{4} \int_{\mathbb{R}^{4}}\left(\exp \left(2 t^{2} u^{2}\right) 2 t^{2} u^{2}-\left(\exp \left(2 t^{2} u^{2}\right)-1\right)\right) d x \\
& <\frac{\lambda}{4} \int_{\mathbb{R}^{4}}\left(\exp \left(2 u^{2}\right) 2 u^{2}-\left(\exp \left(2 u^{2}\right)-1\right)\right) d x \\
& \leq \lim _{k \rightarrow \infty} \frac{\lambda}{4} \int_{\mathbb{R}^{4}}\left(\exp \left(2 u_{k}^{2}\right) 2 u_{k}^{2}-\left(\exp \left(2 u_{k}^{2}\right)-1\right)\right) d x=m_{\lambda}
\end{aligned}
$$


which is a contradiction.

Lemma 6.6. The case $l=0$ cannot occur.

Proof. We prove this by contradiction. If $l=0$, then $u=0$, and $u_{k} \rightarrow 0$ in $L_{l o c}^{2}\left(\mathbb{R}^{4}\right)$. We first claim that:

$$
\int_{\mathbb{R}^{4}}(\gamma-V(x))\left|u_{k}\right|^{2} d x=0 .
$$

For any fixed $\varepsilon>0$, we take $R_{\varepsilon}>0$ such that

$$
|\gamma-V(x)| \leq \varepsilon, \text { for any }|x|>R_{\varepsilon} .
$$

Combining this and the boundedness of $u_{k}$ in $H^{2}\left(\mathbb{R}^{4}\right)$, we derive that

$$
\begin{aligned}
\int_{\mathbb{R}^{4}}(\gamma-V(x))\left|u_{k}\right|^{2} d x & =\int_{B_{R_{\varepsilon}}}(\gamma-V(x))\left|u_{k}\right|^{2} d x+\int_{B_{R_{\varepsilon}}^{c}}(\gamma-V(x))\left|u_{k}\right|^{2} d x \\
& \leq c \int_{B_{R_{\varepsilon}}}\left|u_{k}\right|^{2} d x+\gamma M \varepsilon,
\end{aligned}
$$

where $M=\sup _{k \rightarrow \infty} \int_{\mathbb{R}^{4}}\left|u_{k}\right|^{2} d x$. This together with $u_{k} \rightarrow 0$ in $L_{l o c}^{2}\left(\mathbb{R}^{4}\right)$ as $k \rightarrow \infty$ yields that

$$
\int_{\mathbb{R}^{4}}(\gamma-V(x))\left|u_{k}\right|^{2} d x \leq c \varepsilon
$$

which implies (6.8) holds.

By (6.8), we see that

$$
\lim _{k \rightarrow \infty} \int_{\mathbb{R}^{4}}\left(\left|\Delta u_{k}\right|^{2}+V(x)\left|u_{k}\right|^{2}\right) d x=\int_{\mathbb{R}^{4}}\left(|\Delta u|^{2}+\gamma|u|^{2}\right) d x .
$$

From the proof of Lemma 6.2, we know that there exists some bounded sequence $t_{k} \geq 1$ such that $t_{k} u_{k} \in \mathcal{N}_{\infty}$, that is,

$$
\int_{\mathbb{R}^{4}}\left(\left|\Delta u_{k}\right|^{2}+(\gamma-\lambda)\left|u_{k}\right|^{2}\right) d x-\lambda \int_{\mathbb{R}^{4}}\left(\exp \left(2 t_{k}^{2} u_{k}^{2}\right)-1\right) u_{k}^{2} d x=0 .
$$

On the other hand, since $u_{k} \in N_{V}$, then

$$
\int_{\mathbb{R}^{4}}\left(\left|\Delta u_{k}\right|^{2}+(V(x)-\lambda)\left|u_{k}\right|^{2}\right) d x-\lambda \int_{\mathbb{R}^{4}}\left(\exp \left(2 u_{k}^{2}\right)-1\right) u_{k}^{2} d x=0 .
$$

Combining (6.9) and (6.10), we get

$$
\begin{aligned}
\lambda & \int_{\mathbb{R}^{4}}\left(\exp \left(2 t_{k}^{2} u_{k}^{2}\right)-1\right) u_{k}^{2} d x-\lambda \int_{\mathbb{R}^{4}}\left(\exp \left(2 u_{k}^{2}\right)-1\right) u_{k}^{2} d x \\
& =\int_{\mathbb{R}^{4}}\left(\left|\Delta u_{k}\right|^{2}+(\gamma-\lambda)\left|u_{k}\right|^{2}\right) d x-\int_{\mathbb{R}^{4}}\left(\left|\Delta u_{k}\right|^{2}+(V(x)-\lambda)\left|u_{k}\right|^{2}\right) d x \\
& =\int_{\mathbb{R}^{4}}(\gamma-V(x))\left|u_{k}\right|^{2} d x \rightarrow 0 .
\end{aligned}
$$


GROUND STATES OF BI-HARMONIC EQUATIONS WITH CRITICAL EXPONENTIAL GROWTH 27

Hence

$$
\int_{\mathbb{R}^{4}}\left(\exp \left(2 t_{k}^{2} u_{k}^{2}\right)-1\right) u_{k}^{2} d x=\int_{\mathbb{R}^{4}}\left(\exp \left(2 u_{k}^{2}\right)-1\right) u_{k}^{2} d x+o_{k}(1) .
$$

Next, we claim that $t_{k} \rightarrow t_{0}=1$ as $k \rightarrow \infty$. We prove this by contradiction. Assume that $t_{0}>1$. We carry out the proof in two cases.

Case 1: There exists some $N \geq 2$ such that

$$
\lim _{k \rightarrow \infty} \int_{\mathbb{R}^{4}} u_{k}^{2 N} d x>0
$$

This will imply that

$$
\begin{aligned}
\int_{\mathbb{R}^{4}}\left(\exp \left(2 t_{k}^{2} u_{k}^{2}\right)-1\right) u_{k}^{2} d x & \geq \int_{\mathbb{R}^{4}}\left(\exp \left(2 t_{0}^{2} u_{k}^{2}\right)-1\right) u_{k}^{2} d x \\
& >\int_{\mathbb{R}^{4}}\left(\exp \left(2 u_{k}^{2}\right)-1\right) u_{k}^{2} d x>0,
\end{aligned}
$$

which is a contradiction with (6.11).

Case 2: For any $N \geq 2$, there holds

$$
\lim _{k \rightarrow \infty} \int_{\mathbb{R}^{4}} u_{k}^{2 N} d x \rightarrow 0
$$

Then, we have

$$
\begin{aligned}
I_{V}\left(u_{k}\right) & =\frac{\lambda}{4} \int_{\mathbb{R}^{4}}\left(\exp \left(2 u_{k}^{2}\right) 2 u_{k}^{2}-\left(\exp \left(2 u_{k}^{2}\right)-1\right)\right) d x \\
& =\int_{\mathbb{R}^{4}}\left(\frac{\left(2 u_{k}^{2}\right)^{2}}{2}+\left(\frac{1}{2}-\frac{1}{3 !}\right)\left(2 u_{k}^{2}\right)^{3}+\left(\frac{1}{3 !}-\frac{1}{4 !}\right)\left(2 u_{k}^{2}\right)^{4}+\ldots+\right. \\
& \left.\left(\frac{1}{(n-1) !}-\frac{1}{n !}\right)\left(2 u_{k}^{2}\right)^{n}+\ldots\right) d x \rightarrow \frac{\lambda}{2} \int_{\mathbb{R}^{4}} \exp \left(2 u_{k}^{2}\right) u_{k}^{2} d x .
\end{aligned}
$$

Thus, it follows that $\frac{\lambda}{2} \int_{\mathbb{R}^{4}} \exp \left(2 u_{k}^{2}\right) u_{k}^{2} d x \rightarrow m_{V}$ as $k \rightarrow+\infty$. Therefore, we conclude that

$$
\begin{aligned}
\int_{\mathbb{R}^{4}}\left(\exp \left(2 t_{k}^{2} u_{k}^{2}\right)-1\right) u_{k}^{2} d x & \geq \int_{\mathbb{R}^{4}}\left(\exp \left(2 t_{0}^{2} u_{k}^{2}\right)-1\right) u_{k}^{2} d x \\
& \geq t_{0}^{2} \int_{\mathbb{R}^{4}}\left(\exp \left(2 u_{k}^{2}\right)-1\right) u_{k}^{2} d x \\
& \rightarrow t_{0}^{2} \frac{2 m_{V}}{\lambda}>\frac{2 m_{V}}{\lambda} \\
& =\lim _{k \rightarrow \infty} \int_{\mathbb{R}^{4}}\left(\exp \left(2 u_{k}^{2}\right)-1\right) u_{k}^{2} d x
\end{aligned}
$$

which contradicts (6.11). Thus the claim is proved. 
Now, by (6.8) we can obtain

$$
\begin{aligned}
m_{\infty} & \leq I_{\infty}\left(t_{k} u_{k}\right) \\
& =\frac{t_{k}^{2}}{2} \int_{\mathbb{R}^{4}}\left(\left|\Delta u_{k}\right|^{2}+V(x)\left|u_{k}\right|^{2}\right) d x-\frac{\lambda}{4} \int_{\mathbb{R}^{4}}\left(\exp \left(2 t_{k}^{2} u_{k}^{2}\right)-1\right) d x \\
& =\frac{t_{k}^{2}}{2} \int_{\mathbb{R}^{4}}\left(\left|\Delta u_{k}\right|^{2}+\gamma\left|u_{k}\right|^{2}\right) d x+\frac{t_{k}^{2} \varepsilon^{2}}{2}-\frac{\lambda}{4} \int_{\mathbb{R}^{4}}\left(\exp \left(2 t_{k}^{2} u_{k}^{2}\right)-1\right) d x \\
& \leq \frac{t_{k}^{2}}{2} \int_{\mathbb{R}^{4}}\left(\left|\Delta u_{k}\right|^{2}+\gamma\left|u_{k}\right|^{2}\right) d x+\frac{t_{k}^{2} \varepsilon^{2}}{2}-\frac{\lambda}{4} \int_{\mathbb{R}^{4}}\left(\exp \left(2 u_{k}^{2}\right)-1\right) d x \\
& \rightarrow m_{V}
\end{aligned}
$$

which contradicts (6.3). This accomplishes the proof of Lemma 6.6.

In the following, we consider the case $0<l<\beta$. If $0<l<\beta$, then $u_{k} \rightarrow u \neq 0$ weakly in $H^{2}\left(\mathbb{R}^{4}\right)$. One can choose an increasing sequence $\left\{R_{j}\right\}_{j} \rightarrow+\infty$ such that

$$
\int_{B_{R_{j}}} \exp \left(2 u^{2}\right) u^{2} d x=l+o_{j}(1),
$$

and

$$
\int_{B_{R_{j}}^{c}} u^{p} d x=o_{j}(1)
$$

for any $1 \leq p<\infty$. We define

$$
C_{j}=B_{R_{j}+1} \backslash B_{R_{j}}=\left\{x \in \mathbb{R}^{4}\left|R_{j} \leq\right| x \mid<R_{j}+1\right\} .
$$

Lemma 6.7. For the $C_{j}$ given above, we have

$$
\int_{C_{j}} \exp \left(2 u_{k}^{2}\right) u_{k}^{2} d x=o_{j}
$$

and

$$
\int_{C_{j}}\left|\Delta u_{k}\right|^{2} d x=o_{j}(1) .
$$

Proof. We prove (6.13) by contradiction. If there exists some subsequence $\left\{j_{i}\right\}_{i}$ of $\{j\}$ such that (6.13)fails, then we must have

$$
\sum_{i=1}^{\infty} \int_{C_{j_{i}}} \exp \left(2 u_{k}^{2}\right) u_{k}^{2} d x=\infty .
$$

However, on the other hand,

$$
\begin{aligned}
\sum_{i=1}^{\infty} \int_{C_{j_{i}}} \exp \left(2 u_{k}^{2}\right) u_{k}^{2} d x & \leq \int_{\mathbb{R}^{4}} \exp \left(2 u_{k}^{2}\right) u_{k}^{2} d x \\
& =\frac{1}{\lambda} \int_{\mathbb{R}^{4}}\left(\left|\Delta u_{k}\right|^{2}+V(x)\left|u_{k}\right|^{2}\right) d x<\infty
\end{aligned}
$$



which arrives at a contradiction. Similarly, one can also prove (6.14).

Lemma 6.8. There holds

$$
\lim _{k \rightarrow \infty} \int_{\mathbb{R}^{4}}\left(\exp \left(2 u_{k}^{2}\right)-1\right) d x=\int_{\mathbb{R}^{4}}\left(\exp \left(2 u^{2}\right)-1\right) d x .
$$

Proof. For any $R>0$, we can write

$$
\begin{aligned}
& \left|\int_{\mathbb{R}^{4}}\left(\exp \left(2 u_{k}^{2}\right)-1\right) d x-\int_{\mathbb{R}^{4}}\left(\exp \left(2 u^{2}\right)-1\right) d x\right| \\
& =\left|\int_{\mathbb{R}^{4} \cap\left\{u_{k}<R\right\}}\left(\exp \left(2 u_{k}^{2}\right)-1\right) d x-\int_{\mathbb{R}^{4} \cap\left\{u_{k}<R\right\}}\left(\exp \left(2 u^{2}\right)-1\right) d x\right| \\
& \quad+\left|\int_{\mathbb{R}^{4} \cap\left\{u_{k} \geq R\right\}}\left(\exp \left(2 u_{k}^{2}\right)-1\right) d x-\int_{\mathbb{R}^{4} \cap\left\{u_{k} \geq R\right\}}\left(\exp \left(2 u^{2}\right)-1\right) d x\right| \\
& =I_{k, R}+I I_{k, R} .
\end{aligned}
$$

A direct application of the dominated convergence theorem leads to $I_{k, R} \rightarrow 0$. For $I I_{k, R}$, we have

$$
\begin{aligned}
\int_{\mathbb{R}^{4} \cap\left\{u_{k} \geq R\right\}}\left(\exp \left(2 u_{k}^{2}\right)-1\right) d x & \leq \frac{1}{R^{2}} \int_{\mathbb{R}^{4} \cap\left\{u_{k} \geq R\right\}} u_{k}^{2}\left(\exp \left(2 u_{k}^{2}\right)-1\right) d x \\
& \leq \frac{1}{R^{2}} \int_{\mathbb{R}^{4}} u_{k}^{2} \exp \left(2 u_{k}^{2}\right) d x \rightarrow 0, \text { as } R \rightarrow \infty
\end{aligned}
$$

where we have used the fact that $\int_{\mathbb{R}^{4}} u_{k}^{2} \exp \left(2 u_{k}^{2}\right) d x$ is bounded. Consequently, $I I_{k, R} \rightarrow 0$, and the proof is finished.

Lemma 6.9. If $\int_{\mathbb{R}^{4}}\left(|\Delta u|^{2}+V(x)|u|^{2}\right) d x>\lambda \int_{\mathbb{R}^{4}} \exp \left(2 u^{2}\right) u^{2} d x$, then

$$
\lim _{k \rightarrow \infty} \int_{B_{R_{j}}} \exp \left(2 u_{k}^{2}\right) u_{k}^{2} d x=\int_{B_{R_{j}}} \exp \left(2 u^{2}\right) u^{2} d x
$$

provided $j$ large enough.

Proof. Obviously,

$$
\liminf _{k} \int_{B_{R_{j}}}\left(\left|\Delta u_{k}\right|^{2}+V(x)\left|u_{k}\right|^{2}\right) d x \geq \int_{B_{R_{j}}}\left(|\Delta u|^{2}+V(x)|u|^{2}\right) d x
$$

through lower semi-continuity. We split the proof into two cases.

Case 1: If

$$
\lim _{k} \int_{B_{R_{j}}}\left(\left|\Delta u_{k}\right|^{2}+V(x)\left|u_{k}\right|^{2}\right) d x=\int_{B_{R_{j}}}\left(|\Delta u|^{2}+V(x)|u|^{2}\right) d x,
$$

then by the Adams' inequality (1.9), we know that for any $p>1$,

$$
\begin{aligned}
& \int_{B_{R_{j}}}\left(\exp \left(2 u_{k}^{2}\right) u_{k}^{2}\right)^{p} d x \leq c \int_{B_{R_{j}}}\left(\exp \left(2 p u_{k}^{2}\right)-1\right) u_{k}^{2 p} d x+\int_{B_{R_{j}}} u_{k}^{2 p} d x \\
& \leq\left(\int_{B_{R_{j}}}\left(\exp \left(4 p u_{k}^{2}\right)-1\right) d x\right)^{1 / 2}\left(\int_{B_{R_{j}}} u_{k}^{4 p} d x\right)^{1 / 2}+\int_{B_{R_{j}}} u_{k}^{2 p} d x<+\infty
\end{aligned}
$$


Therefore,

$$
\lim _{k \rightarrow \infty} \int_{B_{R_{j}}}\left(\exp \left(2 u_{k}^{2}\right) u_{k}^{2}\right) d x=\int_{B_{R_{j}}}\left(\exp \left(2 u^{2}\right) u^{2}\right) d x
$$

Case 2: If $\lim _{k} \int_{B_{R_{j}}}\left(\left|\Delta u_{k}\right|^{2}+V(x)\left|u_{k}\right|^{2}\right) d x>\int_{B_{R_{j}}}\left(|\Delta u|^{2}+V(x)|u|^{2}\right) d x$, we set

$$
\begin{aligned}
& v_{k}=\frac{u_{k}}{\lim _{k} \int_{B_{R_{j}}}\left(\left|\Delta u_{k}\right|^{2}+V(x)\left|u_{k}\right|^{2}\right) d x}, \text { and } \\
& v_{0}=\frac{u}{\lim _{k} \int_{B_{R_{j}}}\left(\left|\Delta u_{k}\right|^{2}+V(x)\left|u_{k}\right|^{2}\right) d x} .
\end{aligned}
$$

We claim that there exists some $q>1$ sufficiently closed to 1 such that

$$
q \lim _{k} \int_{B_{R_{j}}}\left(\left|\Delta u_{k}\right|^{2}+V(x)\left|u_{k}\right|^{2}\right) d x<\frac{16 \pi^{2}}{1-\int_{B_{R_{j}}}\left(\left|\Delta v_{0}\right|^{2}+V(x)\left|v_{0}\right|^{2}\right) d x} .
$$

Indeed, we have

$$
\begin{aligned}
& \int_{B_{R_{j}}}\left(\left|\Delta u_{k}\right|^{2}+V(x)\left|u_{k}\right|^{2}\right) d x \cdot\left(1-\int_{B_{R_{j}}}\left(\left|\Delta v_{0}\right|^{2}+V(x)\left|v_{0}\right|^{2}\right) d x\right) \\
& \leq \int_{\mathbb{R}^{4}}\left(\left|\Delta u_{k}\right|^{2}+V(x)\left|u_{k}\right|^{2}\right) d x-\int_{B_{R_{j}}}\left(|\Delta u|^{2}+V(x)|u|^{2}\right) d x \\
& =\frac{\lambda}{2} \int_{\mathbb{R}^{4}}\left(\exp \left(2 u_{k}^{2}\right)-1\right) d x-\frac{\lambda}{2} \int_{\mathbb{R}^{4}}\left(\exp \left(2 u^{2}\right)-1\right) d x+ \\
& \quad+2 I_{V}\left(u_{k}\right)-2 I_{V}(u)+o_{j}(1) .
\end{aligned}
$$

Since $\int_{\mathbb{R}^{4}}\left(|\Delta u|^{2}+V(x)|u|^{2}\right) d x>\lambda \int_{\mathbb{R}^{4}} \exp \left(2 u^{2}\right) u^{2} d x$, then it follows that

$$
I_{V}(u)>\frac{\lambda}{4} \int_{\mathbb{R}^{4}}\left(\exp \left(2 u^{2}\right) 2 u^{2}-\left(\exp \left(2 u^{2}\right)-1\right)\right) d x>0 .
$$

Combining (6.16) and the fact (Lemma 5.3), we conclude that

$$
\lim _{k \rightarrow \infty} I_{V}\left(u_{k}\right)=m_{V}<m_{\infty}<8 \pi^{2} .
$$

This proves the claim.

By the Concentration-Compactness principle for Adams' inequality on $H^{2}\left(\mathbb{R}^{4}\right)$, there exists some $p_{0}>1$ such that

$$
\sup _{k} \int_{B_{R_{j}}}\left(\exp \left(2 u_{k}^{2}\right)-1\right)^{p_{0}} d x<\infty .
$$

Then it follows that there exists some $\tilde{p}_{0}>1$ such that

$$
\sup _{k} \int_{B_{R_{j}}}\left(\exp \left(2 u_{k}^{2}\right) u_{k}^{2}\right)^{\tilde{p}_{0}} d x<\infty
$$


GROUND STATES OF BI-HARMONIC EQUATIONS WITH CRITICAL EXPONENTIAL GROWTH 31

Therefore, we get

$$
\lim _{k \rightarrow \infty} \int_{B_{R_{j}}}\left(\exp \left(2 u_{k}^{2}\right) u_{k}^{2}\right) d x=\int_{B_{R_{j}}} \exp \left(2 u^{2}\right) u^{2} d x
$$

From above, we can extract a subsequence $u_{k_{j}}$ such that for every $j \in \mathbb{N}$,

$$
\int_{C_{j}} \exp \left(2 u_{k_{j}}^{2}\right) u_{k_{j}}^{2} d x=o_{j}(1), \int_{C_{j}}\left|\Delta u_{k_{j}}\right|^{2} d x=o_{j}(1), \int_{C_{j}} u_{k_{j}}^{2} d x=o_{j}(1)
$$

We take $\left\{u_{k_{j}}\right\}$ as a new minimizing sequence renaming it $\left\{u_{j}\right\}_{j}$.

Now, for every $j$, we define a function $\psi_{j} \in C_{c}^{\infty}\left(\mathbb{R}^{4}\right)$ satisfying $0 \leq \psi_{j}(x) \leq 1, \psi_{j}(x)=$ 1 if $|x| \leq R_{j}, \psi_{j}(x)=0$ if $|x|>R_{j}+1$, and $\left|\nabla \psi_{j}(x)\right|,\left|\Delta \psi_{j}(x)\right| \leq c$ for every $x$. We also define auxiliary functions

$$
u_{j}^{\prime}=\psi_{j} u_{j} \text {, and } u_{j}^{\prime \prime}=\left(1-\psi_{j}\right) u_{j}
$$

Obviously, we have $u_{j}=u_{j}^{\prime}+u_{j}^{\prime \prime}$ for every $j$.

Lemma 6.10. The following properties hold as $j \rightarrow \infty$ :

1. $u_{j}^{\prime} \rightarrow u$ weakly in $H^{2}\left(\mathbb{R}^{4}\right)$, strongly in $L^{p}\left(\mathbb{R}^{4}\right)$ for any $1 \leq p<\infty$, and $u_{j}^{\prime \prime} \rightarrow 0$ weakly in $H^{2}\left(\mathbb{R}^{4}\right)$.

2. There results

$$
\begin{aligned}
& \int_{\mathbb{R}^{4}} \exp \left(2 u_{j}^{2}\right) u_{j}^{2} d x \\
& \quad=\int_{\mathbb{R}^{4}} \exp \left(2\left(u_{j}^{\prime}\right)^{2}\right)\left(u_{j}^{\prime}\right)^{2} d x+\int_{\mathbb{R}^{4}} \exp \left(2\left(u_{j}^{\prime \prime}\right)^{2}\right)\left(u_{j}^{\prime \prime}\right)^{2} d x+o_{j}(1) .
\end{aligned}
$$

3. There results

$$
\begin{aligned}
& \int_{\mathbb{R}^{4}}\left(\left|\Delta u_{j}\right|^{2}+V(x)\left|u_{j}\right|^{2}\right) d x \\
& \quad=\int_{\mathbb{R}^{4}}\left(\left|\Delta u_{j}^{\prime}\right|^{2}+V(x)\left|u_{j}^{\prime}\right|^{2}\right) d x+\int_{\mathbb{R}^{4}}\left(\left|\Delta u_{j}^{\prime \prime}\right|^{2}+V(x)\left|u_{j}^{\prime \prime}\right|^{2}\right) d x+o_{j}(1) .
\end{aligned}
$$


Proof. The first property is obvious, by the defnitions of $u_{j}^{\prime}$ and $u_{j}^{\prime \prime}$. Now, we check the second equality. By (6.13), we derive that

$$
\begin{aligned}
& \int_{\mathbb{R}^{4}} \exp \left(2 u_{j}^{2}\right) u_{j}^{2} d x \\
& =\int_{B_{R_{j}}} \exp \left(2 u_{j}^{2}\right) u_{j}^{2} d x+\int_{C_{j}} \exp \left(2 u_{j}^{2}\right) u_{j}^{2} d x+\int_{B_{R_{j}}^{c}+1} \exp \left(2 u_{j}^{2}\right) u_{j}^{2} d x \\
& =\int_{B_{R_{j}}} \exp \left(2\left(u_{j}^{\prime}\right)^{2}\right)\left(u_{j}^{\prime}\right)^{2} d x+\int_{C_{j}} \exp \left(2 u_{j}^{2}\right) u_{j}^{2} d x+\int_{B_{R_{j}}^{c}+1} \exp \left(2\left(u_{j}^{\prime \prime}\right)^{2}\right)\left(u_{j}^{\prime \prime}\right)^{2} d x \\
& =\int_{\mathbb{R}^{4}} \exp \left(2\left(u_{j}^{\prime}\right)^{2}\right)\left(u_{j}^{\prime}\right)^{2} d x+\int_{\mathbb{R}^{4}} \exp \left(2\left(u_{j}^{\prime \prime}\right)^{2}\right)\left(u_{j}^{\prime \prime}\right)^{2} d x+o_{j}(1) .
\end{aligned}
$$

We now prove the third property. Since $q(x)>0$, direct computation leads to

$$
\begin{aligned}
\int_{\mathbb{R}^{4}} V(x)\left|u_{j}\right|^{2} d x & =\int_{B_{R_{j}}} V(x)\left|u_{j}^{\prime}\right|^{2} d x+\int_{B_{R_{j}}^{c}+1} V(x)\left|u_{j}^{\prime \prime}\right|^{2} d x+\int_{C_{j}} V(x)\left|u_{j}\right|^{2} d x \\
& =\int_{B_{R_{j}}} V(x)\left|u_{j}^{\prime}\right|^{2} d x+\int_{B_{R_{j}}^{c}+1} V(x)\left|u_{j}^{\prime \prime}\right|^{2} d x+o_{j}(1) \\
& =\int_{\mathbb{R}^{4}} V(x)\left|u_{j}^{\prime}\right|^{2} d x+\int_{\mathbb{R}^{4}} V(x)\left|u_{j}^{\prime \prime}\right|^{2} d x+o_{j}(1) .
\end{aligned}
$$

We now only need to show that

$$
\int_{\mathbb{R}^{4}}\left|\Delta u_{j}\right|^{2} d x=\int_{\mathbb{R}^{4}}\left|\Delta u_{j}^{\prime}\right|^{2} d x+\int_{\mathbb{R}^{4}}\left|\Delta u_{j}^{\prime \prime}\right|^{2} d x+o_{j}(1) .
$$

Observing

$$
\begin{aligned}
\int_{\mathbb{R}^{4}}\left|\Delta u_{j}\right|^{2} d x & =\int_{\mathbb{R}^{4}}\left|\Delta u_{j}^{\prime}+\Delta u_{j}^{\prime \prime}\right|^{2} d x \\
& =\int_{\mathbb{R}^{4}}\left|\Delta u_{j}^{\prime}\right|^{2} d x+\int_{\mathbb{R}^{4}}\left|\Delta u_{j}^{\prime \prime}\right|^{2} d x+\int_{\mathbb{R}^{4}} \Delta u_{j}^{\prime} \cdot \Delta u_{j}^{\prime \prime} d x
\end{aligned}
$$

in order to obtain the desired result, we only need to verify that

$$
\int_{\mathbb{R}^{4}} \Delta u_{j}^{\prime} \cdot \Delta u_{j}^{\prime \prime} d x=o_{j}(1)
$$


GROUND STATES OF BI-HARMONIC EQUATIONS WITH CRITICAL EXPONENTIAL GROWTH 33

We can write

$$
\begin{aligned}
\int_{\mathbb{R}^{4}} & \Delta u_{k}^{\prime} \cdot \Delta u_{k}^{\prime \prime} d x \\
= & \int_{\mathbb{R}^{4}}\left(u_{j} \Delta \psi_{j}+u_{j} \Delta \psi_{j}+2 \nabla u_{j} \nabla \psi_{j}\right) \cdot\left(u_{j} \Delta\left(1-\psi_{j}\right)\right. \\
& \left.+\left(1-\psi_{j}\right) \Delta u_{j}+2 \nabla\left(1-\psi_{j}\right) \nabla u_{j}\right) d x \\
= & \int_{\mathbb{R}^{4}}\left(u_{j} \Delta \psi_{j}+u_{j} \Delta \psi_{j}+2 \nabla u_{j} \nabla \psi_{j}\right) \cdot\left(-u_{j} \Delta \psi_{j}+\left(1-\psi_{j}\right) \Delta u_{j}-2 \nabla \psi_{j} \nabla u_{j}\right) d x \\
= & \int_{\mathbb{R}^{4}}\left(-\left|u_{j}\right|^{2}\left|\Delta \psi_{j}\right|^{2}+\left(1-\psi_{j}\right) u_{j} \Delta \psi_{j} \Delta u_{j}-2 u_{j} \Delta \psi_{j} \nabla \psi_{j} \nabla u_{j}\right) d x \\
& +\int_{\mathbb{R}^{4}}\left(-\left|u_{j}\right|^{2}\left|\Delta \psi_{j}\right|^{2}+\left(1-\psi_{j}\right) u_{j} \Delta u_{j} \Delta \psi_{j}-2 u_{j} \nabla u_{j} \nabla \psi_{j} \Delta \psi_{j}\right) d x \\
& +\int_{\mathbb{R}^{4}}\left(-2 \nabla u_{j} \nabla \psi_{j} u_{j} \Delta \psi_{j}+2 \nabla u_{j} \nabla \psi_{j}\left(1-\psi_{j}\right) \Delta u_{j}-4\left|\nabla u_{j}\right|^{2}\left|\nabla \psi_{j}\right|^{2}\right) d x \\
= & I+I I+I I I .
\end{aligned}
$$

For $I$, we have

$$
\begin{aligned}
I & =\int_{\mathbb{R}^{4}}\left|\left(-\left|u_{j}\right|^{2}\left|\Delta \psi_{j}\right|^{2}+\left(1-\psi_{j}\right) u_{j} \Delta \psi_{j} \Delta u_{j}-2 u_{j} \Delta \psi_{j} \nabla \psi_{j} \nabla u_{j}\right)\right| d x \\
& \leq c \int_{C_{j}}\left|u_{j}\right|^{2} d x+\int_{C_{j}}\left|u_{j}\right|\left|\Delta u_{j}\right| d x+c \int_{C_{j}}\left|u_{j} \nabla u_{j}\right| d x \leq \\
& \leq c \int_{C_{j}}\left|u_{j}\right|^{2} d x+\left(\int_{C_{j}}\left|u_{j}\right|^{2} d x\right)^{1 / 2}\left(\int_{C_{j}}\left|\Delta u_{j}\right|^{2} d x\right)^{1 / 2} \\
& +c\left(\int_{C_{j}}\left|u_{j}\right|^{2} d x\right)^{1 / 2}\left(\int_{C_{j}}\left|\nabla u_{j}\right|^{2} d x\right)^{1 / 2}=o_{j}(1) .
\end{aligned}
$$

For $I I$, we derive that

$$
\begin{aligned}
I I & =\int_{\mathbb{R}^{4}}\left|\left(-\left|u_{j}\right|^{2}\left|\Delta \psi_{j}\right|^{2}+\left(1-\psi_{j}\right) u_{j} \Delta u_{j} \Delta \psi_{j}-2 u_{j} \nabla u_{j} \nabla \psi_{j} \Delta \psi_{j}\right)\right| d x \\
& \leq \int_{\mathbb{R}^{4}}\left|u_{j}\right|^{2}\left|\Delta \psi_{j}\right|^{2} d x+\int_{\mathbb{R}^{4}}\left|u_{j} \Delta u_{j} \Delta \psi_{j}\right| d x+\int_{\mathbb{R}^{4}}\left|2 u_{j} \nabla u_{j} \nabla \psi_{j} \Delta \psi_{j}\right| d x \\
& \leq c \int_{C_{j}}\left|u_{j}\right|^{2} d x+c\left(\int_{C_{j}}\left|u_{j}\right|^{2} d x\right)^{1 / 2}\left(\int_{C_{j}}\left|\Delta u_{j}\right|^{2} d x\right)^{1 / 2} \\
& +c\left(\int_{C_{j}}\left|u_{j}\right|^{2} d x\right)^{1 / 2}\left(\int_{C_{j}}\left|\nabla u_{j}\right|^{2} d x\right)^{1 / 2}=o_{j}(1) .
\end{aligned}
$$

For $I I I$, obviously we have 


$$
\begin{aligned}
I I I & =\int_{\mathbb{R}^{4}}\left(-2 \nabla u_{j} \nabla \psi_{j} u_{j} \Delta \psi_{j}+2 \nabla u_{j} \nabla \psi_{j}\left(1-\psi_{j}\right) \Delta u_{j}-4\left|\nabla u_{j}\right|^{2}\left|\nabla \psi_{j}\right|^{2}\right) d x \\
& =2 \int_{\mathbb{R}^{4}}\left|\nabla u_{j} \nabla \psi_{j} u_{j} \Delta \psi_{j}\right| d x+2 \int_{\mathbb{R}^{4}}\left|\nabla u_{j} \nabla \psi_{j}\left(1-\psi_{j}\right) \Delta u_{j}\right| d x+4 \int_{\mathbb{R}^{4}}\left|\nabla u_{j}\right|^{2}\left|\nabla \psi_{j}\right|^{2} d x \\
& \leq c\left(\int_{C_{j}}\left|u_{j}\right|^{2} d x\right)^{1 / 2}\left(\int_{C_{j}}\left|\nabla u_{j}\right|^{2} d x\right)^{1 / 2}+c\left(\int_{C_{j}}\left|\nabla u_{j}\right|^{2} d x\right)^{1 / 2} .
\end{aligned}
$$

$$
\cdot\left(\int_{C_{j}}\left|\Delta u_{j}\right|^{2} d x\right)^{1 / 2}+c \int_{C_{j}}\left|\nabla u_{j}\right|^{2} d x .
$$

By using the Sobolev interpolation inequality, we get

$$
\int_{C_{j}}\left|\nabla u_{j}\right|^{2} d x \leq c\left(\int_{C_{j}}\left|u_{j}\right|^{2} d x+\int_{C_{j}}\left|\Delta u_{j}\right|^{2} d x\right)=o_{j}(1) .
$$

Combining (6.19)-(6.21), we finish the proof.

Lemma 6.11. There holds

$$
\frac{\lambda}{4} \int_{\mathbb{R}^{4}}\left(\exp \left(2 u^{2}\right) 2 u^{2}-\left(\exp \left(2 u^{2}\right)-1\right)\right) d x \leq m_{V} .
$$

Proof. Since $u_{j} \rightarrow u$ weakly in $H^{2}\left(\mathbb{R}^{4}\right)$ and in $L^{p}\left(\mathbb{R}^{4}\right)$ for any $p>1$, thus

$$
\begin{aligned}
\frac{\lambda}{4} & \int_{\mathbb{R}^{4}}\left(\exp \left(2 u^{2}\right) 2 u^{2}-\left(\exp \left(2 u^{2}\right)-1\right)\right) d x \\
& \leq \frac{\lambda}{4} \lim _{j \rightarrow+\infty} \int_{\mathbb{R}^{4}}\left(\exp \left(2 u_{j}^{2}\right) 2 u_{j}^{2}-\left(\exp \left(2 u_{j}^{2}\right)-1\right)\right) d x \\
& =m_{V}
\end{aligned}
$$

Lemma 6.12. It cannot be

$$
\int_{\mathbb{R}^{4}}\left(|\Delta u|^{2}+V(x)|u|^{2}\right) d x<\lambda \int_{\mathbb{R}^{4}} \exp \left(2 u^{2}\right) u^{2} d x .
$$

Proof. If (6.22) is true, then there exists some $t \in(0,1)$ such that $t u \in \mathcal{N}_{V}$. Therefore,

$$
\begin{aligned}
m_{V} & \leq I_{V}(t u)=\frac{\lambda}{4} \int_{\mathbb{R}^{4}}\left(\exp \left(2 t^{2} u^{2}\right) 2 t^{2} u^{2}-\left(\exp \left(2 t^{2} u^{2}\right)-1\right)\right) d x \\
& \leq \frac{\lambda}{4} \int_{\mathbb{R}^{4}}\left(\exp \left(2 u^{2}\right) 2 u^{2}-\left(\exp \left(2 u^{2}\right)-1\right)\right) d x \\
& <I_{V}(u) \leq m_{V},
\end{aligned}
$$

which is a contradiction. 
GROUND STATES OF BI-HARMONIC EQUATIONS WITH CRITICAL EXPONENTIAL GROWTH 35

Lemma 6.13. It cannot be

$$
\int_{\mathbb{R}^{4}}\left(|\Delta u|^{2}+V(x)|u|^{2}\right) d x>\lambda \int_{\mathbb{R}^{4}} \exp \left(2 u^{2}\right) u^{2} d x
$$

Proof. By Lemma 6.10, we get

$$
\begin{aligned}
& \int_{\mathbb{R}^{4}}\left(\left|\Delta u_{j}^{\prime}\right|^{2}+V(x)\left|u_{j}^{\prime}\right|^{2}\right) d x+\int_{\mathbb{R}^{4}}\left(\left|\Delta u_{j}^{\prime \prime}\right|^{2}+V(x)\left|u_{j}^{\prime \prime}\right|^{2}\right) d x \\
& =\int_{\mathbb{R}^{4}}\left(\left|\Delta u_{j}\right|^{2}+V(x)\left|u_{j}\right|^{2}\right) d x+o_{j}(1)=\lambda \int_{\mathbb{R}^{4}} \exp \left(2 u_{j}^{2}\right) u_{j}^{2} d x+o_{j}(1) \\
& =\lambda \int_{\mathbb{R}^{4}} \exp \left(2\left(u_{j}^{\prime}\right)^{2}\right)\left(u_{j}^{\prime}\right)^{2} d x+\lambda \int_{\mathbb{R}^{4}} \exp \left(2\left(u_{j}^{\prime \prime}\right)^{2}\right)\left(u_{j}^{\prime \prime}\right)^{2} d x+o_{j}(1) .
\end{aligned}
$$

Assume for contradiction that (6.23) holds, then we can pick some $\delta>0$ such that

$$
\int_{\mathbb{R}^{4}}\left(|\Delta u|^{2}+V(x)|u|^{2}\right) d x>\lambda \int_{\mathbb{R}^{4}} \exp \left(2 u^{2}\right) u^{2} d x+\delta .
$$

Since $u_{j}^{\prime} \rightarrow u$ weakly in $H^{2}\left(\mathbb{R}^{4}\right)$, by (6.23) and Lemma 6.9, we have

$$
\begin{aligned}
& \liminf _{k \rightarrow+\infty} \int_{\mathbb{R}^{4}}\left(\left|\Delta u_{j}^{\prime}\right|^{2}+V(x)\left|u_{j}^{\prime}\right|^{2}\right) d x \\
& \geq \int_{\mathbb{R}^{4}}\left(|\Delta u|^{2}+V(x)|u|^{2}\right) d x \\
& >\lambda \int_{\mathbb{R}^{4}} \exp \left(2 u^{2}\right) u^{2} d x+\delta \\
& =\lambda \int_{\mathbb{R}^{4}} \exp \left(2\left(u_{j}^{\prime}\right)^{2}\right)\left(u_{j}^{\prime}\right)^{2} d x+\delta+o_{j}(1) .
\end{aligned}
$$

Hence, we have

$$
\int_{\mathbb{R}^{4}}\left(\left|\Delta u_{j}^{\prime \prime}\right|^{2}+V(x)\left|u_{j}^{\prime \prime}\right|^{2}\right) d x<\lambda \int_{\mathbb{R}^{4}} \exp \left(2\left(u_{j}^{\prime \prime}\right)^{2}\right)\left(u_{j}^{\prime \prime}\right)^{2} d x-\delta+o_{j}
$$

for $j$ large enough. Since $u_{j}^{\prime \prime} \rightarrow 0$, weakly in $H^{2}\left(\mathbb{R}^{4}\right)$, and arguing as Lemma 6.6, we can obtain

$$
\lim _{j \rightarrow+\infty} \int_{\mathbb{R}^{4}}\left(\left|\Delta u_{j}^{\prime \prime}\right|^{2}+V(x)\left|u_{j}^{\prime \prime}\right|^{2}\right) d x=\int_{\mathbb{R}^{4}}\left(\left|\Delta u_{j}^{\prime \prime}\right|^{2}+\gamma\left|u_{j}^{\prime \prime}\right|^{2}\right) d x .
$$

Therefore, it follows that for $j$ large enough, there holds

$$
\int_{\mathbb{R}^{4}}\left(\left|\Delta u_{j}^{\prime \prime}\right|^{2}+\gamma\left|u_{j}^{\prime \prime}\right|^{2}\right) d x<\lambda \int_{\mathbb{R}^{4}} \exp \left(2\left(u_{j}^{\prime \prime}\right)^{2}\right)\left(u_{j}^{\prime \prime}\right)^{2} d x-\delta+o_{j}(1) .
$$


By the usual argument, we can find some $t_{j} \in(0,1)$ such that $t_{j} u_{j}^{\prime \prime} \in \mathcal{N}_{\infty}$, so we conclude that

$$
\begin{aligned}
m_{\infty} \leq & I_{\infty}\left(t_{j} u_{j}^{\prime \prime}\right) \leq I_{\infty}\left(u_{j}^{\prime \prime}\right) \\
= & \frac{\lambda}{4} \int_{\mathbb{R}^{4}}\left(\exp \left(2\left|u_{j}^{\prime \prime}\right|^{2}\right) 2\left|u_{j}^{\prime \prime}\right|^{2}-\left(\exp \left(2\left|u_{j}^{\prime \prime}\right|^{2}\right)-1\right)\right) d x \\
\leq & \frac{\lambda}{4} \int_{\mathbb{R}^{4}}\left(\exp \left(2\left|u_{j}^{\prime \prime}\right|^{2}\right) 2\left|u_{j}^{\prime \prime}\right|^{2}-\left(\exp \left(2\left|u_{j}^{\prime \prime}\right|^{2}\right)-1\right)\right) d x \\
& +\frac{\lambda}{4} \int_{\mathbb{R}^{4}}\left(\exp \left(2\left|u_{j}^{\prime}\right|^{2}\right) 2\left|u_{j}^{\prime}\right|^{2}-\left(\exp \left(2\left|u_{j}^{\prime}\right|^{2}\right)-1\right)\right) d x \\
\leq & I_{V}\left(u_{j}\right)+o_{j}(1) .
\end{aligned}
$$

Let $j \rightarrow \infty$, we derive $m_{\infty} \leq m_{V}$, which is a contradiction. This accomplishes the proof of Lemma 6.13.

\section{REFERENCES}

[1] S. Adachi, K. Tanaka, Trudinger type inequalities in $\mathbb{R}^{N}$ and their best exponents, Proc. Amer. Math. Soc., 128 (2000), 2051-2057.

[2] D.R. Adams, A sharp inequality of J. Moser for higher order derivatives, Ann. of Math., (2) 128 (1988), 385-398.

[3] Adimurthi, Existence of positive solutions of the semilinear Dirichlet problem with critical growth for the $n$-Laplacian, Ann. Sc. Norm. Sup. Pisa, XVII (1990), 393-413.

[4] F. V. Atkinson, L. A. Peletier, Ground states and Dirichlet problems for $-\Delta u=f(u)$ in $R^{2}$, Arch. Rational Mech. Anal., 96 (1986), 147-165.

[5] F. V. Atkinson, L. A. Peletier, Ground states of $-\Delta u=f(u)$ and the Emden-Fowler equation, Arch. Rational Mech. Anal., 93 (1986), 103-127.

[6] C. Alves, M. Souto, M. Montenegro, Existence of a ground state solution for a nonlinear scalar field equation with critical growth, Calc. Var. Partial Differential Equations, 43 (2012), 537-554.

[7] C. Alves, G. Figueiredo, On multiplicity and concentration of positive solutions for a class of quasilinear problems with critical exponential growth in $\mathbb{R}^{2}$, J. Differential Equations, 246 (2009), 1288-1311.

[8] A. Ambrosetti, P. H. Rabinowitz, Dual variational methods in critical point theory and applications, J. Funct. Anal., 14 (1973), 349-381.

[9] A. Ambrosetti, M. Badiale and S. Cingolani, Semiclassical States of Nonlinear Schrodinger Equations, Arch. Rational Mech. Anal., 140 (1997), 285-300.

[10] A. Ambrosetti and A. Malchiodi, Perturbation methods and semilinear elliptic problems on $R^{n}$. Progress in Mathematics, 240. Birkhuser Verlag, Basel, 2006.

[11] A. Ambrosetti, A. Malchiodi and S. Secchi, Multiplicity results for some nonliear Schrodinger Equations with potentials, Arch. Rational Mech. Anal., 159 (2001), 253-271.

[12] J. Bao, N. Lam and G. Lu, polyharmonic equations with critical exponential growth in the whole space $\mathbb{R}^{n}$, Discrete and Continuous dynamical Systems, 36 (2016), 577-600.

[13] H. Brezis and L. Nirenberg, Positive solutions of nonlinear elliptic problems involving critical Sovolev exponents, Comm. Pure Appl. Math., 36 (1983), 437-477.

[14] L. Chen, J. Li, G. Lu and C. Zhang, Sharpened Adams inequality and ground state solutions to the bi-Laplacian equation in $R^{4}$, Adv. Nonlinear Stud. 18 (2018), no. 3, 429-452. 
[15] L. Chen, G. Lu and C. Zhang, Sharp weighted TrudingerMoserAdams inequalities on the whole space and the existence of their extremals, Calc. Var. Partial Differential Equations, 58(4) (2019), $58: 132$.

[16] D. G. de Figueiredo, O. H. Miyagaki and B. Ruf, Elliptic equations in $\mathbb{R}^{2}$ with nonlinearities in the critical growth range, Calc. Var., 3 (1995), 139-153.

[17] W. Ding and W. Ni, On the existence of positive entire solutions of a semilinear elliptic equation. Arch. Rational Mech. Anal. 91 (1986), no. 4, 283-308.

[18] J. M. do Ó, N-Laplacian equations in $\mathbb{R}^{n}$ with critical growth, Abstr. Appl. Anal., 2 (1997), 301-315.

[19] S. Ibrahim, N. Masmoudi and K. Nakanishi, Trudinger-Moser inequality on the whole plane with the exact growth condition, J. Eur. Math. Soc., 17 (2015), 819-835.

[20] X. Kang and J. Wei, On interacting bumps of semi-classical states of nonlinear Schrdinger equations, Adv. Differential Equations 5 (2000), no. 7-9, 899-928.

[21] H. Kozono, T. Sato and H. Wadade, Upper bound of the best constant of a Trudinger-Moser inequality and its application to a Gagliardo-Nirenberg inequality, Indiana Univ. Math. J., 55 (2006), no. $6,1951-1974$.

[22] N. Lam, G. Lu, Existence of nontrivial solutions to polyharmonic equations with subcritical and critical exponential growth, Discrete and Continuous dynamical Systems, 32 (2012), 2187-2205.

[23] N. Lam, G. Lu, Existence and multiplicity of solutions to equations of $n$-Laplacian type with critical exponential growth in $\mathbb{R}^{n}$, J. Funct. Anal., 262 (2012), 1132-1165.

[24] N. Lam, G. Lu, A new approach to sharp Moser-Trudinger and Adams type inequalities: a rearrangement-free argument, J. Differential Equations, 255 (2013), 298-325.

[25] N. Lam and G. Lu, Sharp Moser-Trudinger inequality on the Heisenberg group at the critical case and applications, Adv. Math., 231 (2012), 3259-3287.

[26] N. Lam and G. Lu, Sharp Adams type inequalities in Sobolev spaces $W^{m, \frac{n}{m}}\left(\mathbb{R}^{n}\right)$ for arbitrary integer $m$, J. Differential Equations, 253 (2012), 1143-1171.

[27] N. Lam, G. Lu and H. Tang, Sharp subcritical Moser-Trudinger inequalities on Heisenberg groups and subelliptic PDEs, Nonlinear Anal., 95 (2014), 77-92.

[28] N. Lam, G. Lu and L. Zhang, Equivalence of critical and subcritical sharp Trudinger-Moser-Adams inequalities, Rev. Mat. Iberoam., 33 (2017),1219-1246.

[29] N. Lam, G. Lu and L. Zhang, Sharp singular Trudinger-Moser inequalities under different norms, Advanced Nonlinear Studies, 19 (2) (2019), 2019-2042.

[30] E. Lenzmann, J. Sok, A sharp rearrangement principle in fourier space and symmetry results for PDEs with arbitrary order, arXiv:1805.06294 1 .

[31] J. Li, G. Lu and Q. Yang, Fourier analysis and optimal Hardy-Adams inequalities on hyperbolic spaces of any even dimension, Adv. Math. 333 (2018), 350-385.

[32] Y. X. Li, B. Ruf, A sharp Trudinger-Moser type inequality for unbounded domains in $\mathbb{R}^{n}$, Indiana Univ. Math. J., 57 (2008), 451-480.

[33] P. L. Lions, The concentration-compactness principle in the calculus of variations. The locally compact case, part 1 and 2, Ann. Inst. Henri. Poincaré Anal. Non linéaire., 1 (1984), 109-145.

[34] G. Lu, H. Tang, Sharp Moser-Trudinger inequalities on hyperbolic spaces with exact growth condition, Journal of Geometric Analysis, 26 (2016), 837-857.

[35] G. Lu, H. Tang and M. Zhu, Best Constants for Adams' Inequalities with the Exact Growth Condition in $\mathbb{R}^{n}$, Advanced Nonlinear Studies, 15(4) (2015), 763-788.

[36] G. Lu and J. Wei, On nonlinear Schrödinger equations with totally degenerate potentials. C. R. Acad. Sci. Paris Sr. I Math. 326 (1998), no. 6, 691-696.

[37] G. Lu and Q. Yang, Sharp Hardy-Adams inequalities for bi-Laplacian on hyperbolic space of dimension four. Adv. Math. 319 (2017), 567-598.

[38] N. Masmoudi, F. Sani, Adams' inequality with the exact growth condition in $\mathbb{R}^{4}$, Comm. Pure Appl. Math., 67 (2014), 1307-1335. 
[39] N. Masmoudi, F. Sani, Trudinger-Moser Inequalities with the Exact Growth Condition in $\mathbb{R}^{4}$ and Applications, Comm. Partial Differential Equations., 40 (2015), 1408-1440.

[40] N. Masmoudi, F. Sani, Higher order Adams' inequality with the exact growth condition, Commun. Contemp. Math., 20 (2018), Doi:10.1142/S0219199717500729.

[41] J. Moser, Sharp form of an inequality by N. Trudinger, Indiana Univ. Maths J., 20 (1971), 1077-1092.

[42] T. Ozawa, On critical cases of Sobolev's inequalities, J. Funct. Anal., 127 (1995), 259-269.

[43] P. Rabinowitz, Minimax methods in critical point theory with applications to differential equations, CBMS Reg. Conf. Ser. Math., 65, American Mathematical Society, Providence, RI,1986.

[44] P. Rabinowitz, On a class of nonlinear Schröinger equations, Z. Angew. Math. Phys., 43 (1992), $27-42$.

[45] P. Rabinowitz, Critical point theory and applications to differential equations: a survey, Topological nonlinear analysis, Progr. Nonlinear Differential Equations Appl., vol. 15, Birkhäser Boston, Boston, MA, 1995, pp. 464-513.

[46] B. Ruf, A sharp Trudinger-Moser type inequality for unbounded domains in $\mathbb{R}^{2}$, J. Funct. Analysis, 219 (2004), 340-367.

[47] B. Ruf, F. Sani, Ground states for elliptic equations in $\mathbb{R}^{2}$ with exponential critical growth, In: Geometric Properties for Parabolic and Elliptic PDE's. Springer INdAM Series, 2 (2013). New York: Springer, 251-267.

[48] B. Ruf, F. Sani, Sharp Adams-type inequalities in $\mathbb{R}^{n}$, Trans. Amer. Math. Soc., 365 (2013), 645-670.

[49] F. Sani, A biharmonic equation in $\mathbb{R}^{4}$ involving nonlinearities with critical exponential growth, Communications on Pure \& Applied Analysis, 12 (2013), 405-428.

[50] N. S. Trudinger, On embeddings in to Orlicz spaces and some applications, J. Math. Mech., 17 (1967), 473-484.

[51] X. Wang, On concentration of positive bound states of nonlinear Schrödinger equations, Comm. Math. Phys. 153 (1993), no. 2, 229-244.

[52] Y. Yang, Existence of positive solutions to quasilinear equations with exponential growth in the whole Euclidean space, Journal of Functional Analysis, 262 (2012), 1679-1704.

[53] L. Zhao, Y. Chang, Minimax level estimate for a singular quasilinear polyharmonic equation in $\mathbb{R}^{2 m}$, J. Differential Equations, 254 (2013), 2434-2464.

\section{Lu Chen}

School of Mathematics and Statistics

Beijing Institute of Technology

Beijing 100081, P. R. China

chenlu5818804@163.com

Guozhen Lu

Department of Mathematics

University of Connecticut

Storrs, CT 06269, USA

E-mail: guozhen.lu@uconn.edu

Maochun Zhu

Faculty of Science

Jiangsu University

Zhenjiang, 212013, P. R. China

zhumaochun2006@126.com 
GROUND STATES OF BI-HARMONIC EQUATIONS WITH CRITICAL EXPONENTIAL GROWTH 39

School of Mathematics and Statistics, Beijing Institute of Technology, Beijing 100081, P. R. ChinA

E-mail address: chenlu5818804@163.com

Department of Mathematics, University of Connecticut, Storrs, CT 06269, USA

E-mail address: guozhen.lu@uconn.edu

Faculty of Science, Jiangsu University, Zhenjiang, 212013, P. R. China,

E-mail address: zhumaochun2006@126.com 